\title{
Genus-2 curves and Jacobians with a given number of points
}

\author{
Reinier Bröker, Everett W. Howe, Kristin E. Lauter and Peter Stevenhagen
}

\begin{abstract}
We study the problem of efficiently constructing a curve $C$ of genus 2 over a finite field $\mathbb{F}$ for which either the curve $C$ itself or its Jacobian has a prescribed number $N$ of $\mathbb{F}$-rational points.

In the case of the Jacobian, we show that any 'CM-construction' to produce the required genus- 2 curves necessarily takes time exponential in the size of its input.

On the other hand, we provide an algorithm for producing a genus- 2 curve with a given number of points that, heuristically, takes polynomial time for most input values. We illustrate the practical applicability of this algorithm by constructing a genus- 2 curve having exactly $10^{2014}+9703$ (prime) points, and two genus- 2 curves each having exactly $10^{2013}$ points.

In an appendix we provide a complete parametrization, over an arbitrary base field $k$ of characteristic neither two nor three, of the family of genus- 2 curves over $k$ that have $k$-rational degree-3 maps to elliptic curves, including formulas for the genus- 2 curves, the associated elliptic curves, and the degree-3 maps.

Supplementary materials are available with this article.
\end{abstract}

\section{Introduction}

For an algebraic variety $V$ defined over a finite field $\mathbb{F}$, a fundamental quantity is its number $N=\# V(\mathbb{F})$ of $\mathbb{F}$-rational points. This quantity, which we briefly refer to as the order of $V$ over $\mathbb{F}$, can be found by a finite computation. However, in view of computer calculations and cryptographic applications, the problem of efficiently counting the number of $\mathbb{F}$-rational points of smooth algebraic varieties defined over a finite field has become a topic of intensive research in the last 25 years.

There is a natural inverse to the point counting problem, which is mathematically and cryptographically interesting as well. It is the problem of efficiently constructing, for a given integer $N$, a smooth variety $V$ over a finite field $\mathbb{F}$ such that $V$ has order $N$ over $\mathbb{F}$. Usually, one restricts the class of varieties $V$ under consideration by requiring that $V$ be, for example, a curve of a given genus or a surface of a given type. In all cases, the question can be phrased in two different ways with respect to $\mathbb{F}$. One may either:

(A) take both $N$ and $\mathbb{F}$ as input, and construct a variety $V$ of order $N$ over $\mathbb{F}$; or

(B) take only $N$ as input, and construct $\mathbb{F}$ and a variety $V$ of order $N$ over $\mathbb{F}$.

In the case of curves of genus 1, that is, elliptic curves, it is a major open problem to find an efficient algorithm for Problem A. The main result of [3] is that, at least heuristically, Problem B for elliptic curves admits an efficient solution if the input $N$ is provided to the algorithm in factored form. In this paper, we generalize this result to curves of genus 2. More precisely, Problem B for elliptic curves admits two natural analogues in higher genus, and our Theorems 1.1 and 1.2 show that they give rise to rather different answers.

For a smooth, projective curve $C$ of genus $g$ defined over a finite field $\mathbb{F}$, the Jacobian $J=\operatorname{Jac} C$ of $C$ is a $g$-dimensional abelian variety over $\mathbb{F}$, and $J(\mathbb{F})$ is a finite abelian group. Using a base point in $C(\mathbb{F})$, one may embed $C$ into $J$ under the Abel-Jacobi map. In the elliptic case $g=1$, this leads to an identification of $C$ and $J$, but in higher genus, $C$ is a strict subvariety

Received 11 April 2014; revised 10 August 2014.

2010 Mathematics Subject Classification 14K22 (primary), 11G15, 11G20, 14G15 (secondary). 
of $J$, and $C(\mathbb{F})$ is merely a subset of the group $J(\mathbb{F})$. This leads to two mathematically natural generalizations in genus 2 of the construction problem for elliptic curves:

(1) construct curves of genus 2 of given order; or

(2) construct curves of genus 2 with Jacobians of given order.

From a cryptographic point of view, the second generalization is the relevant one, as current applications use the group $J(\mathbb{F})$ rather than the set $C(\mathbb{F})$. We will consider both generalizations, taken in the setting of Problem B.

In $\S 4$, we consider the problem of efficiently constructing, for a given integer $N$, a finite field $\mathbb{F}$ and a genus- 2 curve $C$ defined over $\mathbb{F}$ such that $J=\operatorname{Jac} C$ has order $N$ over $\mathbb{F}$. In this case, we say that the pair $(C, \mathbb{F})$ realizes $N$. For our purposes, it suffices to consider only quartic pairs $(C, \mathbb{F})$ realizing $N$. These are pairs for which the subring $\mathbb{Z}[\pi] \subset$ End $J$ generated by the Frobenius element $\pi$ in the endomorphism ring of $J$ is an order in a quartic CM-field $K=\mathbb{Q}(\pi)$; this condition is equivalent to the characteristic polynomial of Frobenius for $C$ being irreducible. The justification for restricting to quartic pairs is that nonquartic pairs will only realize a zero-density subset of all possible input values $N$; see Corollary 4.2.

In view of $[3]$, the natural approach to constructing quartic pairs $(C, \mathbb{F})$ realizing $N$ consists in obtaining $C$ as the reduction of a genus- 2 curve $\widetilde{C}$ in characteristic zero with CM by $K$, since the Igusa modular invariants of such $\widetilde{C}$ may be computed by CM-techniques. As we explain at the end of $\S 4$, a so-called $C M$-construction of $(C, \mathbb{F})$ which, in an intermediary step, writes down the Igusa class polynomials of a curve $\widetilde{C}$ in characteristic zero with CM by $K$ that reduces to $C$ over $\mathbb{F}$, is necessarily exponential in $\log \Delta_{K}$, the size of the discriminant $\Delta_{K}$ of $K=\mathbb{Q}(\pi)$; see Corollary 4.8. As a consequence, CM-constructions are only computationally feasible for CM-fields $K$ of small discriminant.

Given $N$, there are, up to isomorphism, only finitely many pairs $(C, \mathbb{F})$ realizing $N$, so we may define the minimal genus-2 Jacobian discriminant $\Delta(N)$ of $N$ as the smallest discriminant of a CM-field $K=\mathbb{Q}(\pi)$ associated to a quartic pair $(C, \mathbb{F})$ realizing $N$. There are two sets of $N$ s for which this definition is not appropriate. The first is the zero-density set of those $N$ that are realized by nonquartic pairs. The second is the set of those $N$ that are not realized at all as orders of genus-2 Jacobians over finite fields. This is also a zero-density set (Theorem 3.1), and conjecturally it is even empty. For $N$ in one of these two very thin sets, we formally put $\Delta(N)=0$. As we are to prove that $\Delta(N)$ tends to be large, this choice only strengthens Theorem 1.1 below.

In the elliptic case $[\mathbf{3}]$, the expected minimal discriminant of the endomorphism algebra of an elliptic curve of order $N$ over a prime field grows, at least heuristically, as $(\log N)^{2}$, and this gives rise to efficient CM-constructions. For genus 2, we prove that this is not the case.

Theorem 1.1. For an integer $N \in \mathbb{Z}_{>0}$, let $\Delta(N)$ be the minimal genus-2 Jacobian discriminant defined above. Then we have

$$
\limsup _{N \rightarrow \infty} \frac{\Delta(N)}{\sqrt{N}}=+\infty
$$

This theorem implies that any genus-2 CM-construction for abelian surfaces over finite fields of prescribed order $N$, with 'CM-construction' taken in the sense explained above, will have a worst-case run time that is exponential in the input size $\log N$. Section 4 , which contains the proof of Theorem 1.1, provides an explicit worst-case lower bound for the run time of genus-2 CM-constructions solving our problem (Corollary 4.8), as well as a strengthening of the theorem (Corollary 4.6) that shows that the growth of the lim sup does not come from a thin set of $N$ s, and that $\Delta(N) / \sqrt{N}$ becomes in fact large for 'most' $N$.

The proof of Theorem 1.1 is based on a 'scarcity' of small quartic CM-fields $K$ that contain the Weil numbers corresponding to pairs $(C, \mathbb{F})$ realizing $N$. The difference with the genus-1 
situation lies in the fact that the cardinality of the base field $\mathbb{F}$ for genus-2 Jacobians of order $N$ is about $\sqrt{N}$, and not $N$. If one requires the curve $C$ itself to have order $N$ over $\mathbb{F}$, this problem disappears, and one can hope that, just as in the elliptic case, efficient constructions can be given.

The current state of our knowledge on gaps between prime numbers does not allow us to prove that an elliptic curve or genus- 2 curve of order $N$ over a finite field exists for all $N$, but, heuristically and in computational practice, this is never a problem. In $\S 6$, we provide an algorithm that efficiently finds genus- 2 curves of order $N \not \equiv 1 \bmod 6$ in the following sense.

TheOREM 1.2. There exists an algorithm that, on input of an integer $N \not \equiv 1 \bmod 6$ together with its factorization, tries to return a prime number $p$ and a genus-2 curve $C / \mathbb{F}_{p}$ of order $\# C\left(\mathbb{F}_{p}\right)=N$. If there exists an ordinary elliptic curve of order $N$ over a prime field $\mathbb{F}_{p}$ such that $p \equiv N-1 \bmod \ell$ for $\ell=2$ or $\ell=3$, then the algorithm will be successful.

Under standard heuristic assumptions, the required elliptic curve exists for all $N \in \mathbb{Z}_{>1}$, and the expected run time of the algorithm is polynomial in $2^{\omega(N)} \log N$. Here $\omega(N)$ denotes the number of distinct prime factors of $N$.

Although the run time in Theorem 1.2 is not polynomial in the usual sense, it is polynomial in $\log N$ outside a zero-density subset of $\mathbb{Z}_{\geqslant 1}$ consisting of very smooth input values $N$.

The hypothesis on the existence of an elliptic curve of order $N$ in Theorem 1.2 is caused by the fact that we construct the curve $C$ in the theorem as a genus-2 curve with split Jacobian $J \sim E_{1} \times E_{2}$, and this requires the construction of auxiliary elliptic curves $E_{1}$ and $E_{2}$ of given orders. Such elliptic curves can be constructed by the method of [3] discussed in $\S 2$. The Jacobian $J$ of $C$ is then obtained by gluing $E_{1}$ and $E_{2}$ along their $n$-torsion for some integer $n>1$. In this case, the genus- 2 curve $C$ has the special property that it allows nonconstant maps to the elliptic curves $E_{1}$ and $E_{2}$. For $n \leqslant 4$ this is a classical topic, at least when performed over the complex numbers. It was used in the nineteenth century by Jacobi [12], Goursat [8], and others to express hyperelliptic integrals in terms of elliptic integrals.

In $\S 5$ we give an algorithmic description of the gluing results for $n=2$ and $n=3$ that keeps track of fields of definition. For $n=3$ we actually do a little more, in an appendix to the paper: we provide a complete parametrization of the genus- 2 curves that admit rational degree-3 maps to an elliptic curve, including formulas for the genus- 2 curves, the associated elliptic curves, and the degree-3 maps. The explicit gluing algorithms are used in the proof of Theorem 1.2 in $\S 6$. One result of the restriction to $n \in\{2,3\}$ is the congruence condition $N \not \equiv 1 \bmod 6$ in Theorem 1.2. To handle arbitrary $N$ by our method, one would need to use explicit algorithms for gluing two elliptic curves along their $\ell$-torsion for arbitrary primes $\ell$, because only primes $\ell$ coprime to $N-1$ can be used.

In the final $\S 7$, we illustrate the explicit working of our algorithm and construct two genus-2 curves of smooth order $10^{2013}$ and one of prime order $10^{2014}+9703$. The construction of the prime-order curve required finding a root, in a large finite field, of a class polynomial for an imaginary quadratic order of large discriminant. We thank Andrew Sutherland for his generous help in carrying out this calculation for us, using the methods of [24].

In this paper, we view all varieties as being schemes over a given base field. It follows that morphisms of varieties are morphisms over that base field. For example, what we call the endomorphism ring of an abelian surface over a field $k$, other authors might call the ring of $k$-rational endomorphisms.

\section{Elliptic curves of given order}

We start with a review of the elliptic case. Even though Theorem 1.1 expresses the fact that this case is rather different from the genus- 2 case, the elliptic case is used in an essential way in $\S 6$, in the proof of Theorem 1.2. 
For an elliptic curve $E$ defined over a finite field $\mathbb{F}_{q}$ of $q$ elements, the order $N=\# E\left(\mathbb{F}_{q}\right)$ is an integer in the Hasse interval

$$
\mathcal{H}_{q}=\left[(\sqrt{q}-1)^{2},(\sqrt{q}+1)^{2}\right]=[q+1-2 \sqrt{q}, q+1+2 \sqrt{q}]
$$

of length $4 \sqrt{q}$ centered around $q+1$. Note that $N$ and $q$ are of the same size, and that we have a symmetric relation

$$
N \in \mathcal{H}_{q} \Longleftrightarrow q \in \mathcal{H}_{N}
$$

The integers contained in the union of the intervals $\mathcal{H}_{q}$ for those fields $\mathbb{F}_{q}$ that are not prime fields form a zero-density subset of $\mathbb{Z}_{>0}$, so any algorithm realizing elliptic curves of arbitrary prescribed order $N$ can safely restrict to prime fields $\mathbb{F}_{p}$. It is well known that every integer $N \in \mathcal{H}_{p}$ is realized by an elliptic curve over $\mathbb{F}_{p}$, but unfortunately it is unproved that the union $\bigcup_{p \text { prime }} \mathcal{H}_{p}$ of the Hasse intervals for prime fields contains all positive integers. The problem here is that it is unknown whether we have an upper bound

$$
d_{n}=p_{n+1}-p_{n}<4 \sqrt{p_{n}}
$$

for the prime gap $d_{n}$ following the $n$th prime $p_{n}$. This is the bound that makes $\mathcal{H}_{p_{n}}$ and $\mathcal{H}_{p_{n+1}}$ overlap, and that would prevent integers from being outside the Hasse intervals $\mathcal{H}_{p}$ for all primes $p$. Currently, the best proven upper bound [1] is $d_{n}<p_{n}^{0.525}$, which is not good enough for our purposes.

It is possible to prove that only a very thin set of integers $N$ lies outside all Hasse intervals. By a result of Matomäki (see Lemma 3.4), the total length $\sum_{n} d_{n}$ of prime gaps $d_{n}>\sqrt{p_{n}}$ for the primes $p_{n}<X$ is no more than $O\left(X^{2 / 3}\right)$, and this yields an upper bound for the number of integers up to $X$ that are not the order of the group of points of an elliptic curve over a finite field.

Even though (2.3) is unproved, we know by the prime number theorem that, on average, we have $d_{n} \approx \log p_{n}$. This means that finding prime fields over which $N$ can be realized as the order of an elliptic curve is never a practical problem. As the expected number of possible $p$ for a large value $N$ is expected to be about $4 \sqrt{N} / \log N$, there is ample choice in practice.

The key problem arising in the elliptic case is that, given an integer $N \in \mathcal{H}_{p}$, the best general algorithm we know to construct an elliptic curve over $\mathbb{F}_{p}$ of order $N$ is the rather naïve method of picking random elliptic curves over $\mathbb{F}_{p}$ and checking whether their order equals $N$, until a curve of order $N$ is found. As checking the order (and even complete point counting) for elliptic curves over $\mathbb{F}_{p}$ can be done in time polynomial in $\log p \approx \log N$, the run time for this naïve probabilistic algorithm is essentially determined by the number of elliptic curves one has to try before one of order $N$ is encountered. This expected number is of order $\sqrt{N}$, and the resulting run time $O\left(N^{1 / 2+\varepsilon}\right)$ for any $\varepsilon>0$ is exponential in $\log N$, the size of the input value $N$. This means that we do not obtain an efficient algorithm to solve Problem A from the Introduction in the case of elliptic curves.

The solution provided in [3] to construct elliptic curves of prescribed order over a given finite field uses a deterministic complex multiplication approach, which has an even slower run time $O\left(N^{1+\varepsilon}\right)$ for most values of $N$ and $p$. However, it runs in polynomial time in cases where the curve to be constructed has a 'small' endomorphism ring. Heuristically, suitable small endomorphism rings can always be found in the less restrictive setting of Problem B, where, on input $N$, one is free to choose a prime field $\mathbb{F}=\mathbb{F}_{p}$.

To make this more precise, we recall that for an elliptic curve $E$ over $\mathbb{F}_{p}$, the Frobenius endomorphism $\Phi_{p} \in$ End $E$ satisfies a quadratic equation

$$
\Phi_{p}^{2}-t \Phi_{p}+p=0 \in \text { End } E
$$

of discriminant $\Delta=t^{2}-4 p<0$. The associated Weil $p$-polynomial

$$
f=T^{2}-t T+p \in \mathbb{Z}[T]
$$


which may be viewed as the characteristic polynomial of $\Phi_{p}$ acting on the Tate module $T_{\ell}(E)$ of $E$ at a prime $\ell \neq p$, characterizes the isogeny class of $E$, and the elliptic curves in this isogeny class are those elliptic curves over $\mathbb{F}_{p}$ that have order $f(1)=p+1-t$.

To construct, for a given Weil $p$-polynomial $f=T^{2}-t T+p$ of discriminant $\Delta$, an elliptic curve $E$ in the corresponding isogeny class, one can use the complex multiplication method, which realizes $E$ as the reduction modulo $p$ of an elliptic curve in characteristic zero. More precisely, there are only finitely many isomorphism classes of complex elliptic curves with endomorphism ring isomorphic to the imaginary quadratic order $\mathcal{O}_{\Delta}=\mathbb{Z}[T] /(f)$. Complex analytically, these curves arise as quotients $\mathbb{C} / I$ for invertible ideals $I \subseteq \mathcal{O}_{\Delta} \subset \mathbb{C}$. Their $j$-invariants depend only on the class of the ideal $I$ in the class group $\mathrm{Cl}_{\Delta}$, and they are algebraic integers that form the zeros of the Hilbert class polynomial

$$
P_{\Delta}=\prod_{[I] \in \mathrm{Cl} \mathcal{O}_{\Delta}}(T-j(I)) \in \mathbb{Z}[T] .
$$

The polynomial $P_{\Delta}$ splits into distinct linear factors in $\mathbb{F}_{p}[T]$, and its roots in $\mathbb{F}_{p}$ are the $j$-invariants of the elliptic curves over $\mathbb{F}_{p}$ that have Weil polynomial $f$. It is trivial to write down an explicit model $E / \mathbb{F}_{p}$, say in Weierstrass form, with given $j$-invariant $j(E) \in \mathbb{F}_{p}$. The $j$-invariant $j(E)$ determines $E$ up to twists over $\mathbb{F}_{p}$ and, for $\Delta<-4$, the Weil polynomial $f=T^{2} \pm t T+p$ of $E$ up to the sign of $t$. As it is easy to check which of the twists of $E$ has the desired order $N$, finding $j(E) \in \mathbb{F}_{p}$ solves our problem.

The polynomial $P_{\Delta}$ can be used to write down an elliptic curve over $\mathbb{F}_{p}$ of order $N$ if there exist elements $\nu$ and $\pi$ in the imaginary quadratic order $\mathcal{O}_{\Delta}$ satisfying

$$
\nu \bar{\nu}=N, \quad \nu+\pi=1, \quad \pi \bar{\pi}=p \text { (prime) } .
$$

Note that, despite the symmetry in $N$ and $p$, this is just a way to phrase the fact that $N$ is the norm $\operatorname{Norm}(1-\pi)$ for a Weil $p$-number $\pi \in \mathcal{O}_{\Delta}$. As the degree of $P_{\Delta}$, and heuristically also the size of the coefficients of $P_{\Delta}$, are of order of magnitude $|\Delta|^{1 / 2}$, the time needed to compute $P_{\Delta}$ is exponential in $\log |\Delta|$. One therefore looks for the minimal imaginary quadratic order $\mathcal{O}_{\Delta}$ in which there exist elements $\nu$ and $\pi$ satisfying equation (2.6). This order can in principle be found by factoring $N$ in all possible ways as $N=\nu \bar{\nu}$ in $\mathcal{O}_{\Delta}$ for ascending values of $|\Delta|$, until an element $\nu$ is found for which $\pi=1-\nu$ has prime norm $p$. It is explained in [3] how this can be done efficiently if the prime factorization of $N$ in $\mathbb{Z}$ is known and why, on input $N$, the expected minimal value of $|\Delta|$ for which $\pi$ is found is heuristically of size $O\left((\log N)^{2}+2^{\omega(N)}\right)$. Here $\omega(N)$ denotes the number of distinct prime factors of $N$. For our purposes, it suffices to know that the CM-construction of elliptic curves we sketched yields the following result.

Lemma 2.1. The CM-construction produces an elliptic curve $E$ over a prime field $\mathbb{F}_{p}$ that solves Problem B from the Introduction for factored input values $N$ in a time that is heuristically polynomial in $2^{\omega(N)} \log N$.

For a fixed prime $\ell$, the same holds true under the additional restriction that the prime $p$ be congruent to $N-1$ modulo $\ell$ and that the elliptic curve $E$ be ordinary, provided that $N-1$ is not divisible by $\ell$.

Proof. The first statement is [3, Corollary 4.4]. The proof given there also explicitly formulates the heuristic assumption underlying the analysis in the following way: the elements $\nu$ behave like random quadratic integers of norm $N$, in the sense that the norm $N+1-(\nu+\bar{\nu})$ of $\pi=1-\nu$, which is an integer of the same order of magnitude as $N$, will be prime with frequency $1 / \log N$. This random behavior of $\nu$ will also be reflected in the trace $t=\nu+\bar{\nu}$ of $\nu$ taken modulo $\ell$, provided that we keep in mind that the residue class $(N-1+t \bmod \ell)$ in which we find our prime $p=N-1+t$ has to be invertible modulo $\ell$. Thus, we expect that 
$p \equiv N-1 \bmod \ell$ with probability $1 /(\ell-1)$, provided that $N-1$ is not divisible by $\ell$. For fixed $\ell$, this simply adds a constant factor to the expected running time.

For $p>3$, the added restriction that $E$ be ordinary is equivalent to demanding that $p \neq N-1$. Excluding this single value of $p$ does not change the expected running time of the algorithm.

\section{Genus-2 curves and Jacobians}

Let $q$ be a power of a prime. A polynomial $f \in \mathbb{Z}[T]$ is called a Weil q-polynomial if there is an abelian variety $A$ over $\mathbb{F}_{q}$ such that $f$ is the characteristic polynomial of the Frobenius endomorphism $\Phi_{q} \in$ End $A$ acting on the Tate module $T_{\ell}(A)$ for some prime $\ell \nmid q$. As the complex roots of a Weil $q$-polynomial have absolute value $\sqrt{q}$ and come in $g$ complex conjugate pairs, with $g$ the dimension of $A$, the quartic Weil $q$-polynomials arising in genus 2 have the form

$$
\begin{aligned}
f & =T^{4}-a T^{3}+(b+2 q) T^{2}-a q T+q^{2} \\
& =\left(T^{2}+q\right)^{2}-a T\left(T^{2}+q\right)+b T^{2},
\end{aligned}
$$

with $a, b \in \mathbb{Z}$ satisfying the inequalities

$$
2|a| \sqrt{q}-4 q \leqslant b \leqslant \frac{1}{4} a^{2} \leqslant 4 q .
$$

These inequalities define a wedge-shaped region inside the rectangle in the $(a, b)$ plane defined by $|a| \leqslant 4 \sqrt{q}$ and $|b| \leqslant 4 q$, and it is natural to ask which pairs $(a, b)$ of integers satisfying the inequalities (3.2) come from the Weil $q$-polynomial of an abelian surface, or from the Weil $q$-polynomial of the Jacobian of a genus-2 curve. The Honda-Tate theorem [25, Théorème 1] can be used to determine the pairs $(a, b)$ that come from abelian surfaces, and [11, Theorem 1.2] explains how to determine which $(a, b)$ come from Jacobians of curves. For our purposes, it will be sufficient to note that all pairs of integers $(a, b)$ satisfying the inequalities (3.2) and the coprimality condition $\operatorname{gcd}(b, q)=1$ arise from the coefficients of the Weil $q$-polynomial of an abelian surface over $\mathbb{F}_{q}$-in fact, an ordinary abelian surface.

Let $C$ be a curve of genus 2 defined over $\mathbb{F}_{q}$, and let $J=\operatorname{Jac} C$ be its Jacobian, so that $J$ is an abelian surface defined over $\mathbb{F}_{q}$. Let $f$ be the Weil polynomial of $J$, with coefficients given as in $(3.1)$. The pair $\left(\# C\left(\mathbb{F}_{q}\right), \# J\left(\mathbb{F}_{q}\right)\right)$ of orders over $\mathbb{F}_{q}$ is determined by $f$, and conversely. In concrete terms, we have

$$
\begin{aligned}
& \# C\left(\mathbb{F}_{q}\right)=q+1-a \\
& \# J\left(\mathbb{F}_{q}\right)=f(1)=(q+1)^{2}-a(q+1)+b .
\end{aligned}
$$

It follows that the order $\# J\left(\mathbb{F}_{q}\right)$ lies in the genus-2 Hasse interval

$$
\mathcal{H}_{q}^{(2)}=\left[(\sqrt{q}-1)^{4},(\sqrt{q}+1)^{4}\right]
$$

forming the analogue of (2.1). The interval $\mathcal{H}_{q}^{(2)}$ has length $8 \sqrt{q}(q+1)$ and is centered around $q^{2}+6 q+1$. We have an equivalence

$$
N \in \mathcal{H}_{q}^{(2)} \Longleftrightarrow q \in \mathcal{H}_{\sqrt{N}}=\left[\left(N^{1 / 4}-1\right)^{2},\left(N^{1 / 4}+1\right)^{2}\right]
$$

that is not as symmetric as (2.2) in $N$ and $q$. This is because the order $N$ of an abelian surface over $\mathbb{F}_{q}$ has order of magnitude $q^{2}$, not $q$.

Just as in the elliptic case, the union of the integers in the genus-2 Hasse intervals $\mathcal{H}_{q}^{(2)}$ for the fields $\mathbb{F}_{q}$ that are not prime fields forms a zero-density subset of all positive integers. 
Our inability to prove prime gap bounds as in (2.3) prevents us also in this case from showing that every positive integer arises as the order of an abelian surface over a finite field. However, we can prove with some extra effort that, just as in the elliptic case, the (conjecturally empty) set of integers $N$ that do not arise as the order of an abelian surface forms a very thin subset of all positive integers.

TheOREm 3.1. The set of positive integers $N \leqslant X$ that do not occur as the order of an abelian surface over a finite field has cardinality $O\left(X^{5 / 6}\right)$ for $X \rightarrow \infty$.

The proof relies on a lemma about the central part

$$
\mathcal{C}_{q}^{(2)}=\left[(q+1)^{2}-q^{3 / 2},(q+1)^{2}+q^{3 / 2}\right]
$$

of the genus-2 Hasse interval $\mathcal{H}_{q}^{(2)}$.

Lemma 3.2. If $q$ is prime, then every integer in $\mathcal{C}_{q}^{(2)}$ is of the form $f(1)$ for the Weil $q$-polynomial $f$ of some abelian surface over $\mathbb{F}_{q}$.

Proof. Let $N$ be an integer in $\mathcal{C}_{q}^{(2)}$, and write $N=(q+1)^{2}+m$, so that $|m| \leqslant q^{3 / 2}$. We would like to find integers $a$ and $b$ satisfying the inequalities (3.2) such that we also have $m=-a(q+1)+b$ and $\operatorname{gcd}(b, q)=1$; then the polynomial $f$ defined by equation (3.1) will be the Weil $q$-polynomial of an ordinary abelian surface over $\mathbb{F}_{q}$, and $N=f(1)$.

Define three pairs of integers $\left(a_{0}, b_{0}\right),\left(a_{1}, b_{1}\right)$, and $\left(a_{2}, b_{2}\right)$ by setting

$$
\begin{array}{ll}
a_{0}=-\lfloor m /(q+1)\rfloor, & b_{0}=m+a_{0}(q+1), \\
a_{1}=a_{0}-1, & b_{1}=m+a_{1}(q+1), \\
a_{2}=a_{0}-2, & b_{2}=m+a_{2}(q+1) .
\end{array}
$$

We claim that if $q>7$ then at least one of these pairs $\left(a_{i}, b_{i}\right)$ satisfies the inequalities (3.2) and has $\operatorname{gcd}\left(b_{i}, q\right)=1$.

First note that the inequality $|m| \leqslant q^{3 / 2}$ gives

$$
\begin{aligned}
-q^{1 / 2} & <a_{0}<q^{1 / 2}+1, & 0 & \leqslant b_{0} \leqslant q, \\
-q^{1 / 2}-1 & <a_{1}<q^{1 / 2}, & -q-1 & \leqslant b_{1} \leqslant-1, \\
-q^{1 / 2}-2 & <a_{2}<q^{1 / 2}-1, & -2 q-2 & \leqslant b_{2} \leqslant-q-2 .
\end{aligned}
$$

It is easy to check that if $q>7$ then $\left(a_{1}, b_{1}\right)$ satisfies $(3.2)$, so if $\operatorname{gcd}\left(b_{1}, q\right)=1$ we are done. Thus, to prove our claim we may assume that we are in the case where $\operatorname{gcd}\left(b_{1}, q\right) \neq 1$. Since $q$ is prime, we must have $b_{1}=-q$. Therefore $b_{0}=1$ and $b_{2}=-2 q-1$.

Since $b_{0}=1$ we clearly have $\operatorname{gcd}\left(b_{0}, q\right)=1$. We check that the only way $\left(a_{0}, b_{0}\right)$ will fail to satisfy (3.2) is if $a_{0}^{2}<4$. Thus, if $\left(a_{0}, b_{0}\right)$ does not satisfy the desired conditions, it must be the case that $a_{0} \in\{-1,0,1\}$, from which it follows that $a_{2} \in\{-3,-2,-1\}$. To finish the proof of our claim, we may assume we are in this case.

Since $b_{2}=-2 q-1$ we have $\operatorname{gcd}\left(b_{2}, q\right)=1$, and it is easy to check that when $q>7$ and $\left|a_{2}\right| \leqslant 3$, the pair $\left(a_{2}, b_{2}\right)$ satisfies (3.2). This proves our claim, and shows that the lemma holds for $q>7$.

It remains to verify the lemma for primes $q \leqslant 7$. By hand or machine, it is not hard to check that for all of the relevant values of $N$ it is still the case that one of the pairs $\left(a_{i}, b_{i}\right)$ defined above satisfies the inequalities $(3.2)$ and has $\operatorname{gcd}\left(b_{i}, q\right)=1$, with exactly five exceptions: the cases where $(q, N)$ is one of $(2,10),(3,17),(3,21),(5,43)$, or $(7,73)$. For these cases, we can take $f$ to be the Weil polynomial $x^{4}+x^{3}+2 x^{2}+2 x+4, x^{4}+x^{3}+3 x^{2}+3 x+9, x^{4}+2 x^{3}+3 x^{2}+6 x+9$, $x^{4}+2 x^{3}+5 x^{2}+10 x+25$, or $x^{4}+2 x^{3}+7 x^{2}+14 x+49$, respectively. 
REMARK 3.3. With more effort, one can show that the only prime powers $q$ for which the conclusion of Lemma 3.2 fails to hold are the nonprime prime powers $q \leqslant 81$.

We will also use a slight variant of a result of Matomäki [19].

Lemma 3.4. Let $p_{n}$ denote the $n$th prime number and let $d_{n}=p_{n+1}-p_{n}$ denote the $n$th prime gap. For every $c>1 / \sqrt{2}$ there is a constant $B>0$ such that

$$
\sum_{\substack{d_{n}>c \sqrt{p_{n}} \\ p_{n} \leqslant X}} d_{n}<B X^{2 / 3}
$$

for all $X>0$.

Proof. If the lemma is true for a given value of $c$ then it is true for all larger values, so it suffices to consider the case $c<1$.

Matomäki [19, Theorem 1.1, p. 489] states that there is a constant $A>0$ such that, for all $x$, we have

$$
\sum_{\substack{d_{n} \geqslant \sqrt{x} \\ x \leqslant p_{n} \leqslant 2 x}} d_{n}<A x^{2 / 3} .
$$

Let $b=1 / c^{2}$, and note that $1<b<2$. Suppose that $p_{n} \leqslant X$ satisfies $d_{n}>c \sqrt{p_{n}}$, and let $i$ be the unique nonnegative integer such that $p_{n}$ lies in the half-open interval $I_{i}:=\left((b / 2)^{i+1} X,(b / 2)^{i} X\right]$. Set $x=b^{i} X / 2^{i+1}$, so that $I_{i}=(b x, 2 x]$. Since $p_{n}>b x$, we have $c \sqrt{p_{n}}>\sqrt{x}$, so

$$
\sum_{\substack{d_{n}>c \sqrt{p_{n}} \\ b x \leqslant p_{n} \leqslant 2 x}} d_{n} \leqslant \sum_{\substack{d_{n}>\sqrt{x} \\ b x \leqslant p_{n} \leqslant 2 x}} d_{n} \leqslant \sum_{\substack{d_{n}>\sqrt{x} \\ x \leqslant p_{n} \leqslant 2 x}} d_{n}<A x^{2 / 3} .
$$

The interval $(1, X]$ is the union of the intervals $I_{i}$, so

$$
\sum_{\substack{d_{n}>c \sqrt{p_{n}} \\ p_{n} \leqslant X}} d_{n}<\sum_{i \geqslant 0} A\left(\frac{b^{i}}{2^{i+1}} X\right)^{2 / 3}=B X^{2 / 3},
$$

where $B=A /\left(2^{2 / 3}-b^{2 / 3}\right)$.

Proof of Theorem 3.1. By Lemma 3.2, if $N \leqslant X$ is an integer that is not the order of an abelian surface over a finite field, then $N$ does not satisfy $\left|N-(p+1)^{2}\right|<p^{3 / 2}$ for a single prime $p$. If $p_{n}$ and $p_{n+1}$ are consecutive primes for which we have $\left(p_{n}+1\right)^{2}<N<\left(p_{n+1}+1\right)^{2}$, then $p_{n}<Y:=\sqrt{X}$, and we have

$$
\left(p_{n+1}+1\right)^{2}-\left(p_{n}+1\right)^{2}=\left(p_{n+1}-p_{n}\right)\left(p_{n+1}+p_{n}+2\right)>\left(p_{n+1}\right)^{3 / 2}+p_{n}^{3 / 2} .
$$

It follows that the prime gap $d_{n}$ satisfies

$$
d_{n}>\frac{\left(p_{n+1} / p_{n}\right)^{1 / 2} p_{n+1}+p_{n}}{p_{n+1}+p_{n}+2} \sqrt{p_{n}}>\frac{5}{7} \sqrt{p_{n}} .
$$

The number of $N \leqslant X$ that are not orders of abelian surfaces is therefore at most the total length $\sum d_{n} \cdot\left(p_{n+1}+p_{n}+2\right)$ of those intervals $\left[\left(p_{n}+1\right)^{2},\left(p_{n+1}+1\right)^{2}\right]$ for which $p_{n}<Y$ and $d_{n}>\frac{5}{7} \sqrt{p_{n}}$. 
Lemma 3.4 shows that the sum $\sum d_{n}$ over all $n$ for which $p_{n}<Y$ and $d_{n}>\frac{5}{7} \sqrt{p_{n}}$ is bounded by $O\left(Y^{2 / 3}\right)=O\left(X^{1 / 3}\right)$. The $p_{n}$ are all bounded by $X^{1 / 2}$, so the sum $\sum d_{n} \cdot\left(p_{n+1}+p_{n}+2\right)$ is bounded by $O\left(X^{5 / 6}\right)$.

REMARK 3.5. Just as for elliptic curves, it is a safe conjecture that every positive integer occurs as the order of an abelian surface over a finite field. This may be very hard to prove, but there is no practical obstruction in showing any given integer to be the order of an abelian surface, as it will usually arise as $f(1)$ for many quartic Weil $q$-polynomials $f$.

Any product $f=f_{1} \cdot f_{2}$ of two elliptic Weil $q$-polynomials $f_{i}=T^{2}-t_{i} T+q \in \mathbb{Z}[T]$ is a genus-2 Weil $q$-polynomial. It corresponds to the class of abelian surfaces isogenous to the product $E_{1} \times E_{2}$ of elliptic curves $E_{i}$ with Weil polynomial $f_{i}$. If the Jacobian of a genus-2 curve $C$ is in this class, $C$ is said to have split Jacobian. In this split case, the order of the Jacobian factors as

$$
\# J\left(\mathbb{F}_{q}\right)=\# E_{1}\left(\mathbb{F}_{q}\right) \cdot \# E_{2}\left(\mathbb{F}_{q}\right),
$$

whereas the curve itself has order

$$
\# C\left(\mathbb{F}_{q}\right)=q+1-t_{1}-t_{2} .
$$

The explicit construction of curves $C$ from $E_{1}$ and $E_{2}$ is the topic of $\S 5$.

As for genus 1, it is possible to construct abelian surfaces over $\mathbb{F}_{q}$ with a given quartic Weil $q$-polynomial $f \in \mathbb{Z}[X]$ as Jacobians of explicit genus-2 curves using complex multiplication methods. In the most interesting case where $f$ is irreducible, $K=\mathbb{Q}[X] /(f)$ is a quartic CMfield and $\mathcal{O}=\mathbb{Z}[X] /(f)$ an order in $K$. One then wants to find an abelian surface $A / \mathbb{F}_{q}$ for which the subring $\mathbb{Z}\left[\Phi_{q}\right] \subset$ End $A$ generated by the Frobenius $\Phi_{q}$ of $A$ is isomorphic to $\mathcal{O}$. As in the elliptic case, this is done by considering abelian surfaces over the complex numbers admitting $\mathrm{CM}$ by the order $\mathcal{O}$, and even by the full ring of integers $\mathcal{O}_{K} \supseteq \mathcal{O}$ of $K$. Such complex abelian surfaces are quotients of $\mathbb{C}^{2}$ modulo suitably embedded $\mathcal{O}_{K}$-ideals, and their isomorphism class is characterized by three absolute Igusa invariants, just like the isomorphism class of a complex elliptic curve $\mathbb{C} / I$ is characterized by the absolute $j$-invariant of the lattice $I$.

In the elliptic case, the isomorphism classes of the curves $\mathbb{C} / I$ having $C M$ by an imaginary quadratic order correspond to the ideal classes of that order, and their $j$-invariants form the roots of the Hilbert class polynomial (2.5), which lies in $\mathbb{Z}[X]$. In a similar way, the three Igusa invariants of the relevant $\mathcal{O}_{K}$-ideal classes form the roots of three polynomials $H_{i, K} \in \mathbb{Q}[X]$, $i=1,2,3$. They are known as the Igusa class polynomials of the quartic field $K$, and computing them is the key step in any CM-algorithm.

Once one has found the Igusa class polynomials $H_{i, K}$, one can reduce these modulo $p=\operatorname{char}\left(\mathbb{F}_{q}\right)$ to find the Igusa invariants of abelian surfaces $J$ over $\mathbb{F}_{q}$ having CM by $\mathcal{O}_{K}$. Up to twisting over $\mathbb{F}_{q}$, these have the desired Weil $q$-polynomial. Actual equations of abelian surfaces cannot easily be given, but an algorithm of Mestre [20] allows us to write down an explicit genus- 2 curve $C$ having a Jacobian $J$ with the desired Igusa invariants. This allows us to do actual computations in the group $J\left(\mathbb{F}_{q}\right)$, in terms of divisors on $C$.

There are myriad details that go into a full explanation of the genus-2 CM-method, and of the way one can proceed algorithmically. A detailed account that includes the first complete run time analysis was given by Streng [23]. All we will need in $\S 4$ is that the run time of a CM-algorithm to produce genus- 2 curves $C$ with irreducible Weil polynomial $f \in \mathbb{Z}[X]$ is necessarily exponential in the size $\log \Delta_{K}$ of the discriminant $\Delta_{K}$ of $K=\mathbb{Q}[X] /(f)$. This is because the degree of the Igusa class polynomials that occur in the algorithm grows like a positive power of $\Delta_{K}$, as follows.

Proposition 3.6. The degree $n_{K}$ of the Igusa class polynomials of a quartic CM-field $K$ satisfies $n_{K} \gg \Delta_{K}^{1 / 4-\varepsilon}$ for all $\varepsilon>0$. 
Proof. By [23, Lemma 4.14], the degree $n_{K}$ of the Igusa class polynomials of $K$ is, up to a factor 1 or 2 , equal to the relative class number $h_{K}^{-}=h_{K} / h_{K}^{+}$of $K$. Here $h_{K}$ and $h_{K}^{+}$denote the class numbers of $K$ and its real quadratic subfield $K^{+}$. In [18, Corollaries 29 and 32], we find the Brauer-Siegel type result that the logarithm of $h_{K}^{-}$is asymptotic to $\frac{1}{2} \log \left(\Delta_{K}^{-}\right)$, with $\Delta_{K}^{-}=\Delta_{K} / \Delta_{K}^{+}$the quotient of the discriminants of $K$ and $K^{+}$. As we have $\Delta_{K}=\left(\Delta_{K}^{+}\right)^{2} \cdot M$, with $M \in \mathbb{Z}_{>0}$ the absolute norm of the relative discriminant of $K$ over $K^{+}$, we see that $\Delta_{K}^{-}=\Delta_{K}^{+} \cdot M$ is a divisor of $\Delta_{K}$ exceeding $\Delta_{K}^{1 / 2}$, whence $\left(\Delta_{K}^{-}\right)^{1 / 2} \geqslant \Delta_{K}^{1 / 4}$. The result follows.

\section{Genus-2 Jacobians of given order}

We now give a proof of Theorem 1.1. The statement of the theorem is that, in order to realize all integers $N$ in the interval $[1, X]$ as orders of genus-2 Jacobians over finite fields, we will necessarily encounter Weil polynomials generating quartic CM-fields of discriminant exceeding any prescribed constant multiple of $\sqrt{X}$, provided that $X$ is sufficiently large.

All Weil polynomials in this section will be Weil $q$-polynomials of abelian surfaces, that is, quartic polynomials $f \in \mathbb{Z}[T]$ of the form (3.1) arising as the characteristic polynomial of the Frobenius endomorphism acting on the Tate module of an abelian surface defined over $\mathbb{F}_{q}$. If $f$ is such a Weil polynomial and $f(1)$ an integer in the interval $[1, X]$, then the inequalities $(\sqrt{q}-1)^{4} \leqslant f(1) \leqslant X$ imply that we have a bound

$$
\sqrt{q} \leqslant X^{1 / 4}+1
$$

for the square root of the prime power $q$ involved.

We begin by showing that for large $X$, reducible Weil polynomials only account for very few orders of abelian surfaces in the range $[1, X]$.

Proposition 4.1. The number of positive integers $N \leqslant X$ arising as the value $f(1)$ of a reducible quartic Weil polynomial $f \in \mathbb{Z}[T]$ is $O\left(X^{3 / 4}\right)$ for $X \rightarrow \infty$.

Proof. Suppose that $f \in \mathbb{Z}[T]$ is a reducible quartic Weil $q$-polynomial. Since the real roots of a Weil polynomial occur with even multiplicity, the polynomial $f$ is either equal to a product $f=g_{1} g_{2}$ of two quadratic polynomials $g_{1}, g_{2} \in \mathbb{Z}[T]$ with complex conjugate roots of absolute value $\sqrt{q}$, or it is equal to $\left(x^{2}-q\right)^{2}$, in which case we write $f=g_{1} g_{2}$ with $g_{1}=g_{2}=-x^{2}+q$. In both cases, we see that the value $N=f(1)$ is the product of the integers $g_{1}(1)$ and $g_{2}(1)$ in the elliptic Hasse interval $\mathcal{H}_{q}$ defined in (2.1).

We write $N=g_{1}(1) \cdot g_{2}(1)=(s+t)(s-t)$, with $s=\left(g_{1}(1)+g_{2}(1)\right) / 2$ a half-integer lying in $\mathcal{H}_{q}$, and $t=\left|g_{1}(1)-g_{2}(1)\right| / 2$ a nonnegative half-integer of absolute value at most $2 \sqrt{q}$. By (2.1) and (4.1), the positive integer $2 s$ can be bounded by

$$
2 s \leqslant 2(\sqrt{q}+1)^{2} \leqslant 2\left(X^{1 / 4}+2\right)^{2},
$$

whereas $2 t$ is a nonnegative integer not exceeding $4\left(X^{1 / 4}+1\right)$. As the integers $2 s$ and $2 t$ determine $N$, we see that for every $\varepsilon>0$, no more than $(8+\varepsilon) X^{3 / 4}$ values of $N$ occur in $[1, X]$, when $X$ is sufficiently large.

Corollary 4.2. The integers $N$ arising as the value $f(1)$ of a reducible genus-2 Weil polynomial $f \in \mathbb{Z}[T]$ form a zero-density subset of all positive integers.

We can now focus on irreducible Weil polynomials $f$, which have the property that a root of $f$ generates a quartic CM-field $K=\mathbb{Q}[T] /(f)$ over $\mathbb{Q}$. Given $K$, the number of such $f$ can be bounded in the following way; compare to [5, Proposition 4$]$. 
Proposition 4.3. Let $K$ be a quartic $C M$-field having $w_{K}$ roots of unity, and $q$ a prime power. Then there are at most $2 w_{K}$ irreducible quartic Weil q-polynomials having a root in $K$.

Proof. Let $q$ be a power of a prime $p$, and $\pi \in K$ a quartic Weil $q$-number, that is, an algebraic integer $\pi$ of degree four with $|\varphi(\pi)|=\sqrt{q}$ for all complex embeddings $\varphi: K \rightarrow \mathbb{C}$. Then we have $\pi \bar{\pi}=q$, where $x \mapsto \bar{x}$ denotes conjugation over the maximal real subfield $K^{+}$of $K$. By the Honda-Tate theorem [25], the dimension of the abelian varieties in the isogeny class associated to $\pi$ can be read off from properties of the principal ideal $\mathfrak{a}=(\pi)$, which satisfies $\mathfrak{a} \overline{\mathfrak{a}}=(q)$ and is only divisible by primes lying over $p$. In particular, the conjugacy class of $\pi$ corresponds to an isogeny class of abelian surfaces if and only if we have

$$
\frac{f_{\mathfrak{p}} \operatorname{ord}_{\mathfrak{p}} \mathfrak{a}}{\operatorname{ord}_{\mathfrak{p}} q} \in \mathbb{Z}
$$

for every prime $\mathfrak{p}$ of $K$ lying over $p$. Here $f_{\mathfrak{p}}$ denote the residue class degree of $\mathfrak{p}$.

We first show that there are at most four integral ideals $\mathfrak{a}$ of $K$ satisfying $\mathfrak{a} \overline{\mathfrak{a}}=(q)$ for which (4.2) holds for all primes $\mathfrak{p}$ of $K$ over $p$.

Suppose that $\mathfrak{a}$ is such an ideal. Let $\mathfrak{p}$ be a prime of $K$ lying over $p$. If $\mathfrak{p}=\overline{\mathfrak{p}}$ then the condition $\mathfrak{a} \overline{\mathfrak{a}}=(q)$ shows that $2 \operatorname{ord}_{\mathfrak{p}} \mathfrak{a}=\operatorname{ord}_{\mathfrak{p}} q$, so the order of $\mathfrak{a}$ at $\mathfrak{p}$ is determined. On the other hand, suppose that $\mathfrak{p} \neq \overline{\mathfrak{p}}$. Then $f_{\mathfrak{p}} \leqslant 2$, and from $\mathfrak{a} \overline{\mathfrak{a}}=(q)$ we see that

$$
\operatorname{ord}_{\mathfrak{p}} \mathfrak{a}+\operatorname{ord}_{\bar{p}} \mathfrak{a}=\operatorname{ord}_{\mathfrak{p}} q .
$$

Thus, from (4.2) we see that one of the following is true:

(a) $\operatorname{ord}_{\mathfrak{p}} \mathfrak{a}=0$ and $\operatorname{ord}_{\overline{\mathfrak{p}}} \mathfrak{a}=\operatorname{ord}_{\mathfrak{p}} q$; or

(b) $\operatorname{ord}_{\mathfrak{p}} \mathfrak{a}=\operatorname{ord}_{\mathfrak{p}} q$ and $\operatorname{ord}_{\overline{\mathfrak{p}}} \mathfrak{a}=0$; or

(c) $f_{\mathfrak{p}}=2$ and $\operatorname{ord}_{\mathfrak{p}} \mathfrak{a}=\operatorname{ord}_{\overline{\mathfrak{p}}} \mathfrak{a}=(1 / 2) \operatorname{ord}_{\mathfrak{p}} q$.

In short, there is one possibility for $\operatorname{ord}_{\mathfrak{p}} \mathfrak{a}$ if $\mathfrak{p}$ is ramified or inert in $K / K^{+}$, there are two possibilities for the pair $\left(\operatorname{ord}_{\mathfrak{p}} \mathfrak{a}, \operatorname{ord}_{\overline{\mathfrak{p}}} \mathfrak{a}\right)$ if $\mathfrak{p}$ splits in $K / K^{+}$and lies over a prime of $K^{+}$with residue class field degree 1 , and there are at most three possibilities for the pair $\left(\operatorname{ord}_{\mathfrak{p}} \mathfrak{a}, \operatorname{ord}_{\bar{p}} \mathfrak{a}\right)$ if $\mathfrak{p}$ splits in $K / K^{+}$and lies over a prime of $K^{+}$with residue class field degree 2 . By considering the various ways $p$ can split in $K$, we find that there are at most four possibilities for the vector of valuations of $\mathfrak{a}$ at the primes over $p$, so there are no more than four ideals $\mathfrak{a}$ with $\mathfrak{a} \overline{\mathfrak{a}}=(q)$ and such that (4.2) holds for all primes $\mathfrak{p}$ of $K$ over $p$.

Suppose that such an ideal $\mathfrak{a}$ is generated by a Weil number $\pi_{0}$. If $\mathfrak{a}$ is also generated by another Weil number $\pi$, then $\pi / \pi_{0}$ is a unit of $K$, and $\varphi\left(\pi / \pi_{0}\right)$ lies on the unit circle for all embeddings $\varphi$ of $K$ into the complex numbers. It follows that $\pi=\zeta \pi_{0}$ for some root of unity $\zeta$. Therefore, if $\mathfrak{a}$ can be generated by any Weil numbers at all, it can be generated by exactly $w_{K}$ of them. Thus, the number of Weil $q$-numbers in $K$ is at most $4 w_{K}$.

Suppose that $f$ is an irreducible quartic Weil $q$-polynomial with a root $\pi$ in $K$. Then $\bar{\pi}$ is also a root of $f$ in $K$, and $\pi \neq \bar{\pi}$ because $\pi$ is a root of an irreducible quartic and hence not an element of the real subfield of $K$. Thus, every irreducible quartic Weil $q$-polynomial with a root in $K$ produces at least two distinct Weil numbers in $K$, so the number of such polynomials with roots in $K$ is at most $2 w_{K}$.

Corollary 4.4. Let $K$ be a quartic CM-field. Then the number of irreducible genus-2 Weil $q$-polynomials with a root in $K$ that satisfy the bound

$$
\sqrt{q} \leqslant X^{1 / 4}+1
$$

is at most $50 X^{1 / 2} / \log X$ for $X$ sufficiently large.

Proof. It is easy to see that the number of integers less than $y$ of the form $a^{n}$ with $n>1$ is less than $\sqrt{y} \log _{2} y$. Combining this fact with the prime number theorem, we find that the 
number of prime powers less than $y$ is asymptotic to $y / \log y$. It follows that the number of prime powers $q$ up to $\left(X^{1 / 4}+1\right)^{2}$ is less than $(2+\varepsilon) X^{1 / 2} / \log X$, for $X \gg_{\varepsilon} 0$. From Lemma 4.3 we see that for each of these $q$ there are at most $2 w_{K}$ irreducible quartic Weil $q$-polynomials with a root in $K$. Since $w_{K} \leqslant 12$ for quartic fields $K$, the corollary follows.

Now that we know an upper bound on the number of Weil polynomials 'coming from' a fixed quartic CM-field $K$, we still need a result that expresses the fact that there are not too many quartic CM-fields of small discriminant.

Proposition 4.5. For $B \in \mathbb{Z}_{>0}$ a sufficiently large integer, the number of isomorphism classes of quartic CM-fields of discriminant at most $B$ is bounded by $B$.

Proof. If $K$ is a quartic CM-field, then the Galois group over $\mathbb{Q}$ of its normal closure is the dihedral group $D_{4}$ of order 8 , the cyclic group $C_{4}$ of order 4 , or the Klein four-group $V_{4}=C_{2} \times C_{2}$. It follows from the results of Cohen et al. [4] that the number of isomorphism classes of quartic CM-fields $K$ of discriminant $\Delta_{K} \leqslant B$ with group $D_{4}$ is asymptotically equal to $c \cdot B$, where $c \approx 0.05$ is some explicit real constant. As the number of isomorphism classes of quartic fields $K$ with groups $C_{4}$ and $V_{4}$ and bounded discriminant $\Delta_{K} \leqslant B$ is asymptotically much smaller, and grows $[4, \S 1.1]$ like $c^{\prime} \cdot B^{1 / 2}$ and $c^{\prime \prime} \cdot B^{1 / 2}(\log B)^{2}$ for certain explicit positive constants $c^{\prime}, c^{\prime \prime}$, the result follows.

After these preparations, the proof of Theorem 1.1 is more or less straightforward.

Proof of Theorem 1.1. Suppose that

$$
\limsup _{N \rightarrow \infty} \frac{\Delta(N)}{\sqrt{N}}
$$

assumes a finite value. Then there exists a constant $C>0$ such that have

$$
\Delta(N) \leqslant C \sqrt{N} \text { for all integers } N>0 .
$$

Let $\mathbf{A} \subset \mathbb{Z}_{>0}$ be the subset of integers that either are not the value $f(1)$ of any genus-2 Weil polynomial $f$, or are the value $f(1)$ of a reducible genus- 2 Weil polynomial $f$. Then $\mathbf{A}$ is a zero-density subset by Theorem 3.1 and Corollary 4.2 .

For all integers $N \notin \mathbf{A}$, the minimal discriminant $\Delta(N)$ is the discriminant of a quartic CM-field $\mathbb{Q}[T] /\left(f_{N}\right)$, with $f_{N}$ an irreducible quartic Weil polynomial satisfying $N=f_{N}(1)$. If we take $X$ sufficiently large, then there are $(1-\varepsilon) X$ integers $N$ lying in $[1, X] \backslash \mathbf{A}$, with $\varepsilon>0$ small. Moreover, among the CM-fields $\mathbb{Q}[T] /\left(f_{N}\right)$ that occur for these integers, there will be at least $\frac{1}{50}(1-\varepsilon) X^{1 / 2} \log X$ pairwise nonisomorphic fields, as a single isomorphism class will yield no more than $50 X^{1 / 2} / \log X$ polynomials $f_{N}$ by Corollary 4.4. By Proposition 4.5, we will find values

$$
\Delta(N) \geqslant \frac{1}{50}(1-\varepsilon) X^{1 / 2} \log X
$$

among $N \in[1, X]$ for $X \gg_{\varepsilon} 0$. This contradicts (4.3).

As we state it, Theorem 1.1 leaves open the possibility that there is only a very thin set of integers $N$ on which $\Delta(N) / \sqrt{N}$ is unbounded. This is not the case, however, as an easy adaptation of the proof shows.

Corollary 4.6. Let $S$ be a set of positive integers of positive density. Then the minimal genus-2 Jacobian discriminant in Theorem 1.1 satisfies

$$
\limsup _{N \in S, N \rightarrow \infty} \frac{\Delta(N)}{\sqrt{N}}=+\infty
$$


Proof. For any set $S$ of positive density, the number of pairwise nonisomorphic CM-fields $\mathbb{Q}[T] /\left(f_{N}\right)$ encountered (as in the preceding proof) for $N$ on the set $S \backslash \mathbf{A}$ of positive density will still be at least $c X^{1 / 2} \log X$ for some $c>0$.

REMARK 4.7. The single factor of $\log x$ that makes the proof of Theorem 1.1 work suggests that the theorem is rather sharp, but in reality it is not. In fact, Corollary 4.4 is far from optimal, as is does not take into account that the existence of Weil $q$-numbers in a quartic CM-field $K$ implies not only that the rational prime divisor of $q$ has a certain splitting behavior in $K$, as indicated in the proof of Proposition 4.3, but also that the ideal $\mathfrak{a}$ occurring in the proof is principal. In view of the growth of class numbers with $\Delta_{K}$, these are serious restrictions that we simply disregarded.

By a $C M$-construction for genus-2 Jacobians of prescribed order $N$ we mean any algorithm that, in order to find a curve $C$ over $\mathbb{F}$ with Jacobian of order $N$, writes down ${ }^{\dagger}$ the Igusa class polynomials of a quartic CM-field $K$ such that a curve in characteristic zero with CM by $K$ reduces to $C$ over $\mathbb{F}$. Theorem 1.1 implies that such constructions will necessarily have exponential run time.

Corollary 4.8. Any CM-construction for genus-2 Jacobians of prescribed order $N$ will have an exponential run time, of order at least $N^{1 / 8-\varepsilon}$ for all $\varepsilon>0$.

Proof. As the discriminants of the CM-fields involved grow at least as fast as $\sqrt{N}$, by Theorem 1.1, the Igusa class polynomials involved will be of degree at least $N^{1 / 8-\varepsilon}$ by Proposition 3.6, so writing them down takes at least time $N^{1 / 8-\varepsilon}$.

The lower bound in the corollary is rather weak, as it does not account for the size of the coefficients of Igusa polynomials. These also appear to grow as a positive power of the discriminant [23], but we have no good proven lower bounds for the total length of the coefficients. However, the lower bound on the degree of the Igusa class polynomials that we do use shows that any algorithm that requires writing down even just the reduction of an Igusa class polynomial modulo some auxiliary prime will necessarily take exponential time.

\section{Gluing elliptic curves}

Our construction of genus- 2 curves with a given number of points depends on methods of constructing genus- 2 curves that have Jacobians isogenous to a product of given elliptic curves. In this section we will present two algorithms for producing such curves. The first is simply an algorithmic description of an explicit construction given in [10]; the second is based on an explicit construction given in Appendix A.

As is explained in [7], every genus- 2 curve $C$ with a nonsimple Jacobian arises (perhaps in several ways) from specifying two elliptic curves $E_{1}$ and $E_{2}$, an integer $n>1$, and an isomorphism $\psi: E_{1}[n] \rightarrow E_{2}[n]$ of the $n$-torsion subschemes of $E_{1}$ and $E_{2}$ that is an antiisometry with respect to the Weil pairing. More precisely, there is an isogeny $\varphi$ from $E_{1} \times E_{2}$ to the Jacobian Jac $C$ of $C$ whose kernel is the graph of the isomorphism $\psi$, and the pullback via $\varphi$ of the canonical polarization of Jac $C$ is equal to $n$ times the product polarization on $E_{1} \times E_{2}$. In this situation, we say that $\operatorname{Jac} C$ (or, by an abuse of language that we will find convenient, $C$ itself) is obtained by gluing $E_{1}$ and $E_{2}$ together along their $n$-torsion subgroups via $\psi$.

\footnotetext{
$\dagger$ By 'writing down' a polynomial in $x$ of degree $n$, we mean writing down the coefficient of each monomial $1, x, \ldots, x^{n}$ separately, even the ones that happen to be zero. Thus, in this reckoning, it takes time $n+1$ to write down the polynomial $x^{n}-1$.
} 
The relationship between $C$ and $E_{1}$ and $E_{2}$ can also be summarized by saying that there are minimal degree- $n$ maps $\varphi_{i}: C \rightarrow E_{i}$ such that $\varphi_{2 *} \varphi_{1}^{*}=0$; here minimal means that $\varphi_{i}$ does not factor through a nontrivial isogeny. Given $E_{1}, E_{2}, n, \psi, C$, and $\varphi$ from the preceding paragraph, one obtains $\varphi_{i}$ by composing an embedding of $C$ into its Jacobian with the dual isogeny $\widehat{\varphi}$ : Jac $C \rightarrow E_{1} \times E_{2}$, and then projecting $E_{1} \times E_{2}$ onto $E_{i}$. Conversely, given minimal maps $\varphi_{1}$ and $\varphi_{2}$, one takes $\varphi$ to be the degree- $n^{2}$ isogeny

$$
\varphi_{1}^{*} \times \varphi_{2}^{*}: E_{1} \times E_{2} \rightarrow \operatorname{Jac} C
$$

and notes that the kernel of $\varphi$ is the graph of an anti-isometry $\psi: E_{1}[n] \rightarrow E_{2}[n]$.

Over the complex numbers, the full family of genus- 2 curves arising from the case $n=2$ was given in 1832 by Jacobi ([12] or [13, Volume I, pp. 373-382]) as a postscript to his review of Legendre's Traité des fonctions elliptiques [17], and in 1885 Goursat [8, Exemple II, pp. 155-157] gave a family for $n=3$ that misses only a single curve. We will use more recent references because we must keep track of fields of definition, but the formulas we use can be traced back to these early works.

For our intended applications we will be concerned only with the case of curves over finite fields, but the algorithms will work - and will run in polynomial time -over any field $k$ in which elements can be precisely specified and in which arithmetic can be done in polynomial time. We will use the term computationally amenable to describe such fields $k$. It is easy to see that any finitely-generated extension of a prime field is computationally amenable; the principal examples of such fields that we will have in mind are finite fields and number fields.

In fact, our gluing algorithms are based on solving systems of polynomial equations, so the constructions underlying them work over other fields as well; for example, the complex numbers. We could phrase almost all of our results in terms that Jacobi, Legendre, and Goursat would be familiar with, but since we do want to speak about polynomial-time algorithms, we restrict ourselves to computationally amenable fields.

First we give an algorithm that produces the list of all genus- 2 curves that can be obtained by gluing two given elliptic curves along their 2-torsion subgroups; the algorithm is essentially nothing more than a restatement of [10, Proposition 4]. The statement of the algorithm is simplified by the following notation. Suppose that $\alpha_{1}, \alpha_{2}, \alpha_{3}, \beta_{1}, \beta_{2}, \beta_{3}$ are elements of a field $\ell$ of characteristic not two. Let $f$ and $g$ be the monic cubic polynomials whose roots are the $\alpha_{i}$ and the $\beta_{i}$, respectively. Suppose further that $f$ and $g$ are separable and that the quantity $\alpha_{1}\left(\beta_{3}-\beta_{2}\right)+\alpha_{2}\left(\beta_{1}-\beta_{3}\right)+\alpha_{3}\left(\beta_{2}-\beta_{1}\right)$ is nonzero. Set $\alpha_{i j}=\alpha_{i}-\alpha_{j}$ and $\beta_{i j}=\beta_{i}-\beta_{j}$, and define

$$
\begin{array}{ll}
a_{1}=\alpha_{32}^{2} / \beta_{32}+\alpha_{21}^{2} / \beta_{21}+\alpha_{13}^{2} / \beta_{13}, & a_{2}=\alpha_{1} \beta_{32}+\alpha_{2} \beta_{13}+\alpha_{3} \beta_{21}, \\
b_{1}=\beta_{32}^{2} / \alpha_{32}+\beta_{21}^{2} / \alpha_{21}+\beta_{13}^{2} / \alpha_{13}, & b_{2}=\beta_{1} \alpha_{32}+\beta_{2} \alpha_{13}+\beta_{3} \alpha_{21} .
\end{array}
$$

Let $A=\Delta_{g} a_{1} / a_{2}$ and $B=\Delta_{f} b_{1} / b_{2}$, where $\Delta_{f}$ and $\Delta_{g}$ are the discriminants of $f$ and $g$, respectively. Then we let $h_{\alpha_{1}, \alpha_{2}, \alpha_{3}, \beta_{1}, \beta_{2}, \beta_{3}}$ be the polynomial

$$
-\left(A \alpha_{21} \alpha_{13} x^{2}+B \beta_{21} \beta_{13}\right)\left(A \alpha_{32} \alpha_{21} x^{2}+B \beta_{32} \beta_{21}\right)\left(A \alpha_{13} \alpha_{32} x^{2}+B \beta_{13} \beta_{32}\right) .
$$

\section{Algorithm 5.1.}

Input: Weierstrass models of two elliptic curves $E_{1}$ and $E_{2}$ over a computationally amenable field $k$ of characteristic not two.

Output: The set of genus- 2 curves $C$ over $k$ such that there are degree-2 maps $\varphi_{i}: C \rightarrow E_{i}$ for $i=1$ and $i=2$ with $\varphi_{2 *} \varphi_{1}^{*}=0$. 
1. Initialize $L$ to be the empty list.

2. Write $E_{1}$ and $E_{2}$ as $y^{2}=f$ and $y^{2}=g$, respectively, where $f$ and $g$ are separable monic cubic polynomials in $k[x]$. Let $\Delta_{f}$ and $\Delta_{g}$ denote the discriminants of $f$ and $g$.

3. Compute the splitting fields of $f$ and $g$. If the two fields are not isomorphic to one another as extensions of $k$, output the empty set and stop. Otherwise, set $\ell$ to be the splitting field of $f$ and $g$.

4. Compute the roots $\alpha_{1}, \alpha_{2}, \alpha_{3}$ of $f$ and $\gamma_{1}, \gamma_{2}, \gamma_{3}$ of $g$ in $\ell$.

5. For every permutation $\sigma$ of the set $\{1,2,3\}$, do the following:

(a) Set $\beta_{i}=\gamma_{\sigma(i)}$ for each $i$.

(b) If the quantity $\alpha_{1}\left(\beta_{3}-\beta_{2}\right)+\alpha_{2}\left(\beta_{1}-\beta_{3}\right)+\alpha_{3}\left(\beta_{2}-\beta_{1}\right)$ is nonzero, and if the map $\psi: E_{1}[2](\ell) \rightarrow E_{2}[2](\ell)$ defined by $\left(\alpha_{i}, 0\right) \mapsto\left(\beta_{i}, 0\right)$ is Galois equivariant, append the triple $\left(\beta_{1}, \beta_{2}, \beta_{3}\right)$ to $L$.

6. Output the set of all curves

$$
y^{2}=h_{\alpha_{1}, \alpha_{2}, \alpha_{3}, \beta_{1}, \beta_{2}, \beta_{3}}
$$

for all triples $\left(\beta_{1}, \beta_{2}, \beta_{3}\right)$ in $L$.

TheOREM 5.2. Algorithm 5.1 runs in expected polynomial time and produces correct output. The output list will be nonempty if and only if there is an isomorphism $E_{1}[2] \rightarrow E_{2}$ [2] of group schemes over $k$ that is not the restriction to $E_{1}[2]$ of a geometric isomorphism $E_{1} \rightarrow E_{2}$.

Proof. It is clear that the algorithm runs in expected polynomial time. To show that the output is correct, we must analyze the condition from Step $5(\mathrm{~b})$ that the quantity $\alpha_{1}\left(\beta_{3}-\beta_{2}\right)+\alpha_{2}\left(\beta_{1}-\beta_{3}\right)+\alpha_{3}\left(\beta_{2}-\beta_{1}\right)$ be nonzero. Note that this quantity is equal to the determinant

$$
\left|\begin{array}{lll}
\alpha_{1} & \beta_{1} & 1 \\
\alpha_{2} & \beta_{2} & 1 \\
\alpha_{3} & \beta_{3} & 1
\end{array}\right|,
$$

so it is nonzero if and only if there is no affine transformation taking the $\alpha_{i}$ to the $\beta_{i}$, which is equivalent to the condition that the map $\psi: E_{1}[2](\ell) \rightarrow E_{2}[2](\ell)$ from Step 5(b) is not the restriction to $E_{1}[2](\ell)$ of a geometric isomorphism $E_{1} \rightarrow E_{2}$. Thus, in Step 5, the algorithm enumerates all isomorphisms $E_{1}[2] \rightarrow E_{2}[2]$ of group schemes over $k$ that do not come from geometric isomorphisms $E_{1} \rightarrow E_{2}$. The correctness of the output then follows from [10, Propositions 3 and 4].

REmark 5.3. Suppose we write $E_{1}$ and $E_{2}$ in the form $y^{2}=f$ and $y^{2}=g$ for separable monic cubic polynomials $f$ and $g$ in $k[x]$. Since the characteristic of $k$ is not two, giving an isomorphism between the 2-torsion group schemes $E_{1}[2]$ and $E_{2}[2]$ over $k$ is equivalent to giving a Galois-equivariant bijection between the points of order two on $E_{1}$ and the points of order two on $E_{2}$. To give such a bijection, one simply needs to give a Galois-equivariant bijection between the roots of $f$ and the roots of $g$. Such a bijection exists if and only if the splitting fields of $f$ and $g$ are isomorphic to one another as extensions of $k$. When $k$ is finite, these splitting fields will be isomorphic to one another if and only if $E_{1}$ and $E_{2}$ have the same number of $k$-rational points of order 2. Thus, if Algorithm 5.1 is given two elliptic curves over a finite field that have the same number of rational 2-torsion points and that have different $j$-invariants, the output set will be nonempty. 
Next we give an algorithm for gluing two elliptic curves together along their 3-torsion subgroups. As was the case for the preceding algorithm and its proof, it will be convenient to have some explicit formulas for the family of genus- 2 curves obtained by such 3 -gluings. Such formulas (for part or all of the family of such curves) have appeared in the literature, going back at least to 1876 (see, for example, $[\mathbf{8}, \mathbf{9}, \mathbf{1 6}, \mathbf{2 2}]$ ), but none of the references we have found have all of the information we would like to have about this family of curves. However, using these references, we were able to work out all of the desired details; we have collected our results in Appendix A.

Some notation will be helpful. If $k$ is a field, let $k^{*}$ act on the set of quadruples $(a, b, c, d) \in k^{4}$ by setting

$$
\lambda(a, b, c, d)=\left(\lambda^{2} a, \lambda^{3} b, \lambda^{-2} c, \lambda^{-3} d\right)
$$

for $\lambda \in k^{*}$, and denote the orbit of $(a, b, c, d)$ under this action by $[a: b: c: d]$. We denote the set of these orbits by $P_{k}$.

\section{Algorithm 5.4.}

Input: Weierstrass models of two elliptic curves $E_{1}$ and $E_{2}$ over a computationally amenable field $k$ of characteristic neither two nor three.

Output: The set of genus- 2 curves $C$ over $k$ such that there are degree-3 maps $\varphi_{i}: C \rightarrow E_{i}$ for $i=1$ and $i=2$ with $\varphi_{2 *} \varphi_{1}^{*}=0$.

1. Initialize $L$ to be the empty list.

2. Let $j_{1}$ and $j_{2}$ be the $j$-invariants of $E_{1}$ and $E_{2}$, and define elements of the polynomial ring $k[w, x, y, z]$ as follows:

$$
\begin{aligned}
& g_{1}=1728\left(w^{2} y+4 w x z-4 x^{2} y^{2}\right)^{3}-j_{1}\left(w^{3}+x^{2}\right)^{2}\left(y^{3}+z^{2}\right), \\
& g_{2}=1728\left(w y^{2}+4 x y z-4 w^{2} z^{2}\right)^{3}-j_{2}\left(w^{3}+x^{2}\right)\left(y^{3}+z^{2}\right)^{2}, \\
& g_{3}=12 w y+16 x z-1 .
\end{aligned}
$$

3. Find all elements $[a: b: c: d]$ of $P_{k}$ that satisfy $g_{1}, g_{2}$, and $g_{3}$, and such that either $a b \neq 0$ or $c d \neq 0$.

4. For every $p \in P_{k}$ from Step 3:

(a) Choose $a, b, c, d \in k$ such that $p=[a: b: c: d]$.

(b) If $\left(a^{3}+b^{2}\right)\left(c^{3}+d^{2}\right) \neq 0$, compute representatives $t \in k^{*}$ of the elements of the (finite and possibly empty) set $S \subseteq k^{*} / k^{* 2}$ such that the curves $E_{a, b, c, d, t, 1}$ and $E_{a, b, c, d, t, 2}$ from Appendix A are isomorphic to $E_{1}$ and $E_{2}$. For each such $t$, append the quintuple $(a, b, c, d, t)$ to the list $L$.

5. If $j_{1}=j_{2}=0$, write $E_{1}$ in the form $v^{2}=u^{3}+e_{1}$ and $E_{2}$ in the form $v^{2}=u^{3}+e_{2}$, with $e_{1}, e_{2} \in k$. If $e_{1} e_{2} \in 4 k^{* 6}$, set $b=e_{1}$ and $d=1 /\left(16 e_{1}\right)$, and append the quintuple $(0, b, 0, d, 2)$ to the list $L$.

6. If $j_{1}=j_{2}=1728$, write $E_{1}$ in the form $v^{2}=u^{3}+e_{1} u$ and $E_{2}$ in the form $v^{2}=u^{3}+e_{2} u$, with $e_{1}, e_{2} \in k$. If $e_{1} e_{2} \in 108 k^{* 4}$, set $a=e_{1}$ and $c=1 /\left(12 e_{1}\right)$, and append the quintuple $(a, 0, c, 0,2)$ to the list $L$.

7. If any quintuple in $L$ is equivalent to an earlier quintuple under the action of $k^{*} \times k^{*}$ described in Appendix A, delete the later quintuple from $L$.

8. Output the set of all curves $C_{a, b, c, d, t}$ (from Appendix A) for $(a, b, c, d, t) \in L$. 
ThEOREm 5.5. Algorithm 5.4 runs in expected polynomial time and produces correct output. The output list will be nonempty if the following conditions are satisfied:

(a) there exists an isomorphism $E_{1}[3] \rightarrow E_{2}[3]$ of group schemes that is an anti-isometry with respect to the Weil pairing; and

(b) the curves $E_{1}$ and $E_{2}$ are not 2-isogenous to one another over the algebraic closure of $k$.

Proof. If we show that Step 3 and Step 4(b) can be completed in expected polynomial time, it will be clear that the entire algorithm runs in expected polynomial time.

To begin, we note that an easy calculation shows that $[-1 / 4: 1 / 8:-1:-1]$ is the only element $[a: b: c: d]$ of $P_{k}$ that satisfies $g_{1}=g_{2}=g_{3}=0$ and for which $\left(a^{3}+b^{2}\right)\left(c^{3}+d^{2}\right)=0$.

Suppose that $p=[a: b: c: d]$ is an element of $P_{k}$ such that $g_{1}(p)=g_{2}(p)=g_{3}(p)=0$ and such that $a b \neq 0$. Since $a b \neq 0$, there is a unique representative for $p$ such that $a=b$. With this normalization, we find that $g_{1}=g_{2}=g_{3}=0$ becomes a system of three equations in three unknowns $a, c$, and $d$. Proposition A.2 from the Appendix shows that every solution to this system over $\bar{k}$ with $\left(a^{3}+a^{2}\right)\left(c^{3}+d^{2}\right) \neq 0$ gives a genus- 2 curve $C_{a, a, c, d, 1}$ over $\bar{k}$ along with degree-3 maps $\varphi_{a, a, c, d, 1,1}$ and $\varphi_{a, a, c, d, 1,2}$ to the elliptic curves over $\bar{k}$ with $j$-invariants equal to $j_{1}$ and $j_{2}$, and distinct solutions over $\bar{k}$ give rise to nonisomorphic triples $\left(C, \varphi_{1}, \varphi_{2}\right)$. There are at most 24 such triples [14, Theorem 1], so there are at most 24 solutions to the system over $\bar{k}$ with $\left(a^{3}+a^{2}\right)\left(c^{3}+d^{2}\right) \neq 0$. As we noted above, there is only one solution with $\left(a^{3}+a^{2}\right)\left(c^{3}+d^{2}\right)=0$. Therefore, the variety determined by $g_{1}=g_{2}=g_{3}=0$ and $a=b$ is zero-dimensional, and computing its points over $k$ is an expected polynomial-time computation.

Likewise, in expected polynomial time one can compute the points $p=[a: b: c: d]$ of $P_{k}$ such that $g_{1}(p)=g_{2}(p)=g_{3}(p)=0$ and such that $c d \neq 0$. Thus, Step 3 can be completed in expected polynomial time.

To show that Step 4(b) runs in expected polynomial time, we note that over a field $k$ of characteristic neither two nor three, it is easy to determine the set of $t \in k^{*}$ (modulo $k^{* 2}$ ) such that the quadratic twist of one elliptic curve by $t$ is isomorphic to a second elliptic curve: one simply writes the two curves in short Weierstrass form as $y^{2}=x^{3}+A x+B$ and $y^{2}=x^{3}+A^{\prime} x+B^{\prime}$, and computes the set of $t \in k^{*}$ such that $A^{\prime}=A t^{2}$ and $B^{\prime}=B t^{3}$. Finding these $t$ can clearly be done in expected polynomial time. There is at most one solution $t$ to these equations, unless $A=A^{\prime}=0$ or $B=B^{\prime}=0$. If $A=A^{\prime}=0$ the solutions, if any, all lie in the same class of $k^{*} / k^{* 2}$. If $B=B^{\prime}=0$ there are either zero or two solutions; if there are two solutions, they lie in the same class of $k^{*} / k^{* 2}$ if and only if -1 is a square in $k$. Thus, Step 4(b), and hence the entire algorithm, runs in expected polynomial time.

Next we must show that the output of the algorithm is correct. We see from Proposition A.2 that the set we intend the algorithm to output is equal to the set of all curves $C_{a, b, c, d, t}$ with $\left(a^{3}+b^{2}\right)\left(c^{3}+d^{2}\right) t \neq 0$ and $12 a c+16 a d=1$ such that, for each $i$, we have $E_{a, b, c, d, t, i} \cong E_{i}$.

Certainly every curve $C_{a, b, c, d, t}$ in the set output by the algorithm satisfies $\left(a^{3}+b^{2}\right)\left(c^{3}+d^{2}\right)$ $t \neq 0$ and $12 a c+16 b d=1$, and has the property that $E_{a, b, c, d, t, i} \cong E_{i}$ for each $i$; Step 4(b) explicitly enforces these requirements, and an easy calculation shows that the curves (if any) obtained from Steps 5 and 6 also have these properties.

On the other hand, suppose that $(a, b, c, d, t)$ is a quintuple such that $\left(a^{3}+b^{2}\right)\left(c^{3}+d^{2}\right) t \neq 0$ and $12 a c+16 b d=1$ and such that $E_{a, b, c, d, t, i} \cong E_{i}$ for each $i$. We see from equations (A.2) and (A.3) that since $\left(a^{3}+b^{2}\right)\left(c^{3}+d^{2}\right) t \neq 0$, the equations $g_{1}$ and $g_{2}$ in Step 2 express the condition that the elliptic curves $E_{a, b, c, d, t, i}$ and $E_{i}$ have the same $j$-invariant, for $i=1$ and $i=2$. Thus, Steps 3 and 4 ensure that the algorithm will find $(a, b, c, d, t)$ (up to the action of $\left.k^{*} \times k^{*}\right)$ if $a b \neq 0$ or $c d \neq 0$.

Suppose that our quintuple $(a, b, c, d, t)$ has $a b=0$ and $c d=0$. We see from the condition that $12 a c+16 b d=1$ that then either $a=c=0$ or $b=d=0$. If $a=c=0$, then equations (A.2) 
and (A.3) show that $j_{1}=j_{2}=0$, and we find that $E_{1}$ and $E_{2}$ must be isomorphic to the curves

$$
t y^{2}=x^{3}+512 b^{4} d^{3} \quad \text { and } \quad t y^{2}=x^{3}+512 b^{3} d^{4},
$$

respectively. Using the condition that $16 b d=1$ and rescaling the variables $x$ and $y$, we find that $E_{1}$ and $E_{2}$ are isomorphic to

$$
y^{2}=x^{3}+b t^{3} / 8 \text { and } y^{2}=x^{3}+d t^{3} / 8 .
$$

Thus, the $e_{1}$ and $e_{2}$ from Step 5 must satisfy $e_{1}=b t^{3} r^{6} / 8$ and $e_{2}=d t^{3} s^{6} / 8$ for some $r, s \in k^{*}$. It follows that $e_{1} e_{2} \in 4 k^{* 6}$. Furthermore, given any $e_{1}$ and $e_{2}$ with $e_{1} e_{2} \in 4 k^{* 6}$, if we take $b=e_{1}, d=1 /\left(16 e_{1}\right)$, and $t=2$, then $E_{0, b, 0, d, t, i} \cong E_{i}$ for each $i$.

On the other hand, if $b=d=0$, then equations (A.2) and (A.3) show that $j_{1}=j_{2}=1728$, and we find that $E_{1}$ and $E_{2}$ must be isomorphic to the curves

$$
t y^{2}=x^{3}+36 a^{3} c^{2} \text { and } t y^{2}=x^{3}+36 a^{2} c^{3},
$$

respectively. Using the condition that $12 a c=1$ and rescaling the variables $x$ and $y$, we find that $E_{1}$ and $E_{2}$ are isomorphic to

$$
y^{2}=x^{3}+\left(a t^{2} / 4\right) x \quad \text { and } \quad y^{2}=x^{3}+\left(c t^{2} / 4\right) x
$$

Thus, the $e_{1}$ and $e_{2}$ from Step 6 must satisfy $e_{1}=a t^{2} r^{4} / 4$ and $e_{2}=c t^{2} s^{4} / 4$ for some $r, s \in k^{*}$. It follows that $e_{1} e_{2} \in 108 k^{* 4}$. Furthermore, given any $e_{1}$ and $e_{2}$ with $e_{1} e_{2} \in 108 k^{* 4}$, if we take $a=e_{1}, c=1 /\left(12 e_{1}\right)$, and $t=2$, then $E_{a, 0, c, 0, t, i} \cong E_{i}$ for each $i$.

Thus, every curve $C$ in the list output by the algorithm does have degree-3 maps $\varphi_{i}: C \rightarrow E_{i}$ such that $\varphi_{2 *} \varphi_{1}^{*}=0$, so the output is correct.

Now suppose that conditions (a) and (b) of the theorem hold. Condition (a) says that there is an isomorphism $\psi: E_{1}[3] \rightarrow E_{2}[3]$ of group schemes that is an anti-isometry with respect to the Weil pairing. As is explained in [7], associated to this data there is a (possibly singular) curve $C$ of arithmetic genus 2, together with degree-3 maps $\varphi_{1}: C \rightarrow E_{1}$ and $\varphi_{2}: C \rightarrow E_{2}$ such that $\varphi_{2 *} \varphi_{1}^{*}=0$. Combining condition (b) with a result of Kani [14, Theorem 3], we find that $C$ is in fact a nonsingular curve. Thus $C$ will appear in the list output by the algorithm, so the list is nonempty.

\section{Genus-2 curves of given order}

In this section we prove Theorem 1.2. Our strategy will be to look at curves $C$ over finite prime fields $\mathbb{F}_{p}$ such that the Jacobian $J$ of $C$ is isogenous to a product $E_{1} \times E_{2}$ of elliptic curves. As we noted in equation (3.5) in $\S 3$, if $E_{1}$ and $E_{2}$ have traces $t_{1}$ and $t_{2}$, then $C$ will have $p+1-t_{1}-t_{2}$ rational points. Leaving aside for the moment the question of how to produce $C$ from $E_{1}$ and $E_{2}$, we see that if we are given an integer $N$, we would like to produce a prime $p$ and two elliptic curves $E_{1}$ and $E_{2}$ over $\mathbb{F}_{p}$ with traces that sum to $p+1-N$.

In $\S 1$ we noted the difficulty constructing an elliptic curve over a given finite field with a given trace of Frobenius (Problem A). However, there is an easy special case of this problem. Given a prime $p$, it is very easy to produce a supersingular elliptic curve over $\mathbb{F}_{p}$ (see [2]), and for $p>3$ all such curves have trace 0 . We therefore use the following strategy for producing a curve $C$ with a given number $N$ of points:

- Construct an elliptic curve $E_{1}$ over some prime field $\mathbb{F}_{p}$ such that the trace $t_{1}$ of $E_{1}$ satisfies $t_{1}=p+1-N$; that is, $\# E_{1}\left(\mathbb{F}_{p}\right)=N$.

- Construct a supersingular curve $E_{2}$ over $\mathbb{F}_{p}$, so that the trace $t_{2}$ of $E_{2}$ satisfies $t_{2}=0$. 
- Construct a genus- 2 curve $C$ over $\mathbb{F}_{p}$ whose Jacobian is isogenous to $E_{1} \times E_{2}$. Then from equation (3.5) we find

$$
\# C\left(\mathbb{F}_{p}\right)=p+1-t_{1}-t_{2}=N .
$$

In order to obtain an actual algorithm from this outline, we begin with some results that help us produce elliptic curves to use as input data for Algorithms 5.1 and 5.4. In order to obtain one or more genus- 2 curves from one of these algorithms, the two elliptic curves that are input to the algorithm must have isomorphic $\ell$-torsion subgroup schemes, where $\ell=2$ for Algorithm 5.1 and $\ell=3$ for Algorithm 5.4. Definition 6.1 (below) and the results that follow it help us produce elliptic curves in a given isogeny class whose $\ell$-torsion subgroup schemes have a known structure.

Let $E$ be an elliptic curve over a finite field $k$ of cardinality $q$, and let $\pi$ denote the Frobenius endomorphism of $E$. Suppose that the endomorphism ring of $E$ is an order in an imaginary quadratic field. (This will be the case precisely when the endomorphism ring is commutative, and precisely when $\pi$ does not lie in $\mathbb{Z}$.) Then the ring $\mathbb{Z}[\pi]$ is a subring of finite index in End $E$.

Definition 6.1. Let $\ell$ be a prime. The elliptic curve $E$ is minimal at $\ell$ if the index of $\mathbb{Z}[\pi]$ in End $E$ is not divisible by $\ell$.

Let $\Delta$ be the discriminant of End $E$ and let $t$ be the trace of the Frobenius endomorphism of $E$, so that the discriminant of $\mathbb{Z}[\pi]$ is equal to $t^{2}-4 q$. We see that $E$ is minimal at $\ell$ if and only if $\left(t^{2}-4 q\right) / \Delta$ is not divisible by $\ell$.

Lemma 6.2. Let $E$ be an elliptic curve over a finite field $k$ whose endomorphism ring is commutative, and let $\ell$ be a prime not equal to the characteristic of $k$. Then $E$ is minimal at $\ell$ if and only if the number of $k$-rational rank- $\ell$ subgroup schemes of $E$ is less than $\ell+1$.

Proof. Let $V$ be the group $E[\ell](\bar{k})$, viewed as a two-dimensional $\mathbb{F}_{\ell^{-}}$-vector space. The Frobenius endomorphism $\pi$ of $E$ acts invertibly on $V$, so we can view it as an element $x$ of GL $(V)$. The rank- $\ell$ subgroup schemes of $E$ correspond to one-dimensional eigenspaces of $x$, so there will be $\ell+1$ of these subgroup schemes when $x$ acts as a scalar, and fewer than $\ell+1$ subgroup schemes otherwise. If $x$ acts as multiplication by an integer $a$, then $\pi-a$ kills all of $E[\ell]$, so the endomorphism $\pi-a$ of $E$ factors through multiplication by $\ell$, and $(\pi-a) / \ell$ is an endomorphism $\alpha$ of $E$. Conversely, if $(\pi-a) / \ell$ is an endomorphism of $E$, then $\pi$ acts as a scalar on $E[\ell]$.

Thus, there are $\ell+1 k$-rational rank- $\ell$ subgroup schemes of $E$ precisely when $\pi$ lies in $\mathbb{Z}+\ell$ End $E$, which is the case precisely when the index of $\mathbb{Z}[\pi]$ in End $E$ is divisible by $\ell$.

(In the ordinary case, the lemma also follows from [6, Theorem 2.1, p. 278].)

The next algorithm shows that it is easy to produce curves that are minimal at a given prime.

\section{Algorithm 6.3.}

Input: A triple $(E, H, \ell)$, where $E$ is an ordinary elliptic curve over a finite field $k$ of characteristic greater than three such that End $E$ is a maximal order in a quadratic field, where $H$ is the image in $k[x]$ of the Hilbert class polynomial of this maximal order, and where $\ell$ is an integer equal to either two or three.

Output: An elliptic curve over $k$ that is isogenous to $E$ and that is minimal at $\ell$. 
1. If $E$ has fewer than $\ell+1$ subgroup schemes of rank $\ell$, return $E$ and stop.

2. Choose a rank- $\ell$ subgroup scheme $G$ of $E$ so that the $j$-invariant of the quotient curve $E / G$ is not a root of $H$.

3. Set $E_{0}=E$ and $E_{1}=E / G$, and set $i=1$.

4. If $E_{i}$ has fewer than $\ell+1$ rank- $\ell$ subgroup schemes, return $E_{i}$, and stop.

5. Pick a rank- $\ell$ subgroup scheme $G_{i}$ of $E_{i}$ such that $E_{i} / G_{i}$ is not isomorphic to $E_{i-1}$, and set $E_{i+1}=E_{i} / G_{i}$.

6. Increment $i$, and go to Step 4 .

REMARK 6.4. If we write an elliptic curve $E / k$ as $y^{2}=x^{3}+a x+b$, then the rank-2 subgroup schemes of $E$ correspond to the roots of $x^{3}+a x+b$ in $k$; a root $r$ corresponds to the rank-2 subgroup scheme $G$ that contains the point $(r, 0)$, and the quotient $E / G$ can be written $y^{2}=x^{3}-\left(4 a+15 r^{2}\right) x+(14 a r+22 b)$. The rank-3 subgroup schemes of $E$ correspond to the roots of the 3 -division polynomial $3 x^{4}+6 a x^{2}+12 b x-a^{2}$; a root $r$ corresponds to the rank-3 subgroup scheme $G$ that contains the two geometric points of $E$ with $x$-coordinate equal to $r$, and the quotient $E / G$ can be written $x^{3}-\left(9 a+30 r^{2}\right) x-\left(42 a r+27 b+70 r^{3}\right)$.

TheOREm 6.5. Algorithm 6.3 is correct, and runs in expected polynomial time.

Proof. The algorithm follows a path, without backtracking, along the 'isogeny volcano' of $\ell$-isogenies [6] (see also $[\mathbf{1 5}, \S 4.2]$ ). The curve $E_{0}$ is on the rim of the volcano, and the condition that the $j$-invariant of $E / G$ not be a root of $H$ ensures that $E_{1}$ is not on the rim of the volcano. Therefore the isogeny $E_{0} \rightarrow E_{1}$ is 'descending', and the general theory shows all of the successive isogenies in the path are also descending. The maximal number of steps on the descending path before an $\ell$-minimal curve is reached is the $\ell$-adic valuation of the conductor of the order of discriminant $t^{2}-4 q$, which is polynomial in the input size.

REMARK 6.6. In Algorithm 6.3, we restrict $\ell$ to be two or three merely to avoid a discussion on the representation of subgroup schemes of larger rank.

REMARK 6.7. In general, one can easily produce an $\ell$-minimal curve isogenous to a given $E$, even when End $E$ is not maximal and when no Hilbert class polynomial is provided; one simply traverses three paths starting at $E$, but with different first steps. One of the paths is guaranteed to be descending. However, in our application we will have the Hilbert class polynomial at hand anyway, so we give this slightly simpler algorithm.

Now we reach the algorithm that we will use to prove Theorem 1.2.

\section{Algorithm 6.8 .}

Input: A positive integer $N \not \equiv 1 \bmod 6$ together with its factorization.

Output: A prime $p$ and a genus-2 curve $C$ over $\mathbb{F}_{p}$ such that $\# C\left(\mathbb{F}_{p}\right)=N$, or the word 'failed'.

1. If $N$ is even, set $\ell=2$. Otherwise, set $\ell=3$.

2. Use the modified version of the algorithm of Bröker and Stevenhagen [3] discussed below in Remark 6.9 to try to produce a fundamental discriminant $\Delta$, the Hilbert class polynomial $H$ for $\Delta$, a prime $p>3$ congruent to $N-1$ modulo $\ell$, and an ordinary elliptic curve $E$ over $\mathbb{F}_{p}$ with CM by $\Delta$ and with $\# E\left(\mathbb{F}_{p}\right)=N$. If this step fails, output 'failed' and stop. 
3. Apply Algorithm 6.3 to $E, H$, and $\ell$ to find an elliptic curve $E_{1}$ over $\mathbb{F}_{p}$, isogenous to $E$, that is minimal at $\ell$.

4. Use the algorithm of Bröker [2] to produce a trace-zero elliptic curve $E_{2}$ over $\mathbb{F}_{p}$.

5. If $\ell=2$ do the following:

(a) If $E_{2}$ has three rational points of order 2, replace $E_{2}$ by a 2-isogenous curve that has only one rational point of order 2 .

(b) Apply Algorithm 5.1 to $E_{1}$ and $E_{2}$, choose a curve $C$ from the resulting list, output $p$ and $C$, and stop.

6. If $\ell=3$ do the following:

(a) Apply Algorithm 5.4 to the curves $E_{1}$ and $E_{2}$. If the algorithm returns a nonempty list of curves, choose a curve $C$ from the list, output $p$ and $C$, and stop.

(b) Compute a curve $E_{2}^{\prime}$ that is 2-isogenous to $E_{2}$.

(c) Apply Algorithm 5.4 to the curves $E_{1}$ and $E_{2}^{\prime}$, choose a curve $C$ from the list returned by the algorithm, output $p$ and $C$, and stop.

REMARK 6.9. Recall the outline of the Bröker-Stevenhagen algorithm [3, p. 2168], sketched in $\S 2$. Given a positive integer $N$, together with its factorization, the algorithm will produce a pair $(d, \nu)$, where $d$ is a squarefree positive integer and $\nu$ is an integer of the field $\mathbb{Q}(\sqrt{-d})$ such that $\nu$ has norm $N$ and $1-\nu$ has norm equal to a prime. The algorithm runs by looking at each imaginary quadratic field $K$ in turn, finding all integers $\nu \in K$ of norm $N$, and waiting until one of these $\nu$ satisfies the condition that the norm of $1-\nu$ is prime.

For Step 2 of Algorithm 6.8, we need to use a version of the Bröker-Stevenhagen algorithm modified as follows. The input to the algorithm now includes an auxiliary prime $\ell$. As in the original algorithm, we run through fields $K$ and integers $\nu$ of $K$ with norm $N$ until we find a $\nu$ such that the norm of $1-\nu$ is a prime $p$, but now we add in three additional restrictions:

(i) $p>3$;

(ii) $p \neq N-1$; and

(iii) $p \equiv N-1 \bmod \ell$.

(Given the first condition, the second condition is equivalent to requiring the algorithm to output an ordinary elliptic curve.) As we explained in the proof of Lemma 2.1, this modified algorithm has a heuristic expected running time polynomial in $\ell 2^{\omega(N)} \log N$.

Proof of Theorem 1.2. We will prove Theorem 1.2 by showing that Algorithm 6.8 has the required properties.

Note that if there exist a prime $p$ with $p \equiv N-1 \bmod \ell$ and an ordinary elliptic curve $E$ over $\mathbb{F}_{p}$ with $\# E\left(\mathbb{F}_{p}\right)=N$, then there also exist such a $p$ and $E$ with $p>3$. This is easy to check when $N<8$, and when $N \geqslant 8$ the condition $p>3$ follows from the condition that $\# E\left(\mathbb{F}_{p}\right)=N$.

As we discussed in Remark 6.9, the modified Bröker-Stevenhagen algorithm will succeed in producing an ordinary elliptic curve $E$ over a prime field $\mathbb{F}_{p}$ with $\# E\left(\mathbb{F}_{p}\right)=N$ and with $p \equiv N-1 \bmod \ell$, whenever such a curve exists. Under standard heuristic assumptions, the modified algorithm runs in time polynomial in $\ell 2^{\omega(N)} \log N$, and since $\ell \leqslant 3$ this is also polynomial in $2^{\omega(N)} \log N$. Thus, for the rest of the proof, we may assume that Step 2 succeeds in producing an $E$ over a prime field $\mathbb{F}_{p}$ as above - and, as we noted, we may also assume that $p>3$. Since $E$ is ordinary, it cannot have trace zero.

Step 3 will run in expected polynomial time. 
Bröker's algorithm will produce a supersingular curve $E_{2}$ over $\mathbb{F}_{p}$, and will run in polynomial time if the generalized Riemann hypothesis is true. Thus Step 4 will succeed in polynomial time under standard hypotheses.

Suppose that $N$ is even. Let $\pi$ denote the Frobenius of $E_{2}$, so that $\pi^{2}+p=0$ and the index of the ring $\mathbb{Z}[\pi]$ in End $E_{2}$ is either one or two. It follows that every curve isogenous to $E_{2}$ that is not minimal at two is 2-isogenous to one that is. Since a curve of even order is minimal at two if and only if it has just one rational point of order two, we see that Step 5(a) will succeed.

The curve $E_{1}$ from Step 3 is minimal at two and has an even number $N$ of points, so it also has exactly one rational point of order two. Since $E_{1}$ and $E_{2}$ are defined over a finite field, it follows that the group schemes $E_{1}[2]$ and $E_{2}[2]$ are isomorphic to one another. Also, since $E_{1}$ is ordinary and $E_{2}$ is supersingular, the two curves have different $j$-invariants. Thus, in Step 5(b), Algorithm 5.1 will succeed in producing a genus-2 curve $C$ whose Jacobian is isogenous to $E_{1} \times E_{2}$, so that $C$ will have $N$ points.

Finally, suppose we have reached Step 6, and suppose that in Step 6(a), Algorithm 5.4 fails to return a curve $C$. According to Theorem 5.5, this can only happen if there is not an antiisometry $E_{1}[3] \rightarrow E_{2}[3]$, or if there is a 2-isogeny from $E_{1}$ to $E_{2}$ over the algebraic closure of the base field. However, since $E_{1}$ is ordinary and $E_{2}$ is supersingular, the two curves are not geometrically isogenous to one another, so there is no anti-isometry $E_{1}[3] \rightarrow E_{2}[3]$.

The curve $E_{2}$ has even order, so we can compute a 2 -isogenous curve $E_{2}^{\prime}$ as required by Step 6(b). Recall that $E_{1}$ was constructed to be minimal at three, and note that $E_{2}$ is also minimal at three, because (as we noted earlier) the index of the ring $\mathbb{Z}[\pi]$ in End $E_{2}$ is either one or two. Therefore we can apply [11, Lemma 4.3, p. 249], and we find that either there is an anti-isometry $E_{1}[3] \rightarrow E_{2}[3]$ or there is an anti-isometry $E_{1}[3] \rightarrow E_{2}^{\prime}[3]$. Since there is not one from $E_{1}[3]$ to $E_{2}[3]$, there must be one from $E_{1}[3]$ to $E_{2}^{\prime}[3]$. Combining this with the fact that $E_{1}$ and $E_{2}^{\prime}$ are not geometrically isogenous (because one curve is ordinary and the other supersingular) and applying Theorem 5.5, we find that Algorithm 5.4 applied to $E_{1}$ and $E_{2}^{\prime}$ will produce at least one curve $C$. Therefore, Step 6(c) will succeed.

\section{Explicit examples}

We conclude by explicitly constructing several genus- 2 curves having a prescribed large number of points. The large numbers we chose for our examples are $N_{1}=10^{2013}$ and $N_{2}=10^{2014}+9703$, the smallest prime larger than $10^{2014}$. One of our examples we are able to specify completely here; the equations for the others can be found on the second author's web site, by starting at http://alumni.caltech.edu/ however/biblio.html

and following the link associated with this paper. The Magma sources are also available as online supplementary material from the publisher's website.

\subsection{A genus-2 curve of order $10^{2013}$}

The first step in our construction is to produce an elliptic curve of order $N_{1}=10^{2013}$. As explained in [3], elliptic curves of 10-power order can often be constructed with endomorphism ring $\mathbb{Z}[i]$, the smallest imaginary quadratic order in which either two or five splits completely; the order $\mathbb{Z}[(-1+\sqrt{-31}) / 2]$ of discriminant -31 , in which both two and five split completely, is expected to work in all cases. We will show that both of these orders can be used to produce a curve of order $N_{1}$.

Let $i$ be a square root of -1 , and take

$$
\nu=2^{1006} \cdot 5^{164} \cdot(1+i) \cdot(2+i)^{1685} .
$$

Then $\operatorname{Norm}(\nu)=10^{2013}$ and $p=\operatorname{Norm}(1-\nu)$ is prime, and the elliptic curve $E: y^{2}=x^{3}-x$ over $\mathbb{F}_{p}$ has $10^{2013}$ points. However, this curve is not minimal at two; in fact, the large power of 
two that appears in $\nu$ ensures that the index of $\mathbb{Z}[\pi]$ in End $E$ is divisible by $2^{1006}$. Therefore, to find an isogenous curve $E_{1}$ that is minimal at two, we must travel 1006 steps down a very tall isogeny volcano. This can be done without much trouble, but there is no clear way of expressing the $j$-invariant of the resulting curve in a compact manner.

The prime $p$ is inert in the quadratic field of discriminant -19 , so any curve over $\mathbb{F}_{p}$ with CM by the order of discriminant -19 must be supersingular (and have trace zero). The Hilbert class polynomial for this discriminant is $x+96^{3}$, so we can take $E_{2}$ to be any curve over $\mathbb{F}_{p}$ with $j$-invariant $-96^{3}$. The discriminant of the characteristic polynomial of Frobenius for $E_{2}$ is $-4 p$, which is a fundamental discriminant because $p \equiv 1 \bmod 4$. It follows that $E_{2}$ is minimal at two. Gluing $E_{1}$ and $E_{2}$ together along their 2-torsion subgroups gives us a genus-2 curve $C$ over $\mathbb{F}_{p}$ with $10^{2013}$ points.

We chose our $E_{2}$ so that the curve $C$ that we obtained could be written as $y^{2}=x^{6}+$ $c_{4} x^{4}+c_{2} x^{2}+1$, for certain $c_{2}, c_{4}$ in $K$. This curve has obvious maps to the elliptic curves $y^{2}=x^{3}+c_{4} x^{2}+c_{2} x+1$ and $y^{2}=x^{3}+c_{2} x^{2}+c_{4} x+1$. At the URL mentioned above, we give the values of $c_{2}$ and $c_{4}$, as well as Magma code that shows that the two quotient elliptic curves have the number of points that we claim.

\subsection{Another genus-2 curve of order $10^{2013}$}

To avoid the long chain of 2-isogenies that the preceding construction required, we can replace the order $\mathbb{Z}[i]$ with an order in which two splits, and then require that $\nu$ not be divisible by many powers of two. (We will have to take $\nu$ to be divisible by two, in order for $1-\nu$ to have prime norm.) For this example, we use the order $\mathbb{Z}[\omega]$ of discriminant -31 , where $\omega=(-1+\sqrt{-31}) / 2$. We find that the integer

$$
\nu=2(\omega-1) \cdot 5^{322} \cdot(4 \omega+1)^{456}(\omega+1)^{670}
$$

has norm $10^{2013}$, and $p=\operatorname{Norm}(1-\nu)$ is a 2014-digit prime. If we then take $E$ to be the appropriate twist of an elliptic curve over $\mathbb{F}_{p}$ whose $j$-invariant is a root of the Hilbert class polynomial for discriminant -31 , we will have $\# E\left(\mathbb{F}_{p}\right)=10^{2013}$. For this $E$, we need take only one step down the isogeny volcano to find an isogenous curve $E_{1}$ that is minimal at two. Since $p \equiv 3 \bmod 4$, we can take $E_{2}$ to be the curve $y^{2}=x^{3}+x$. Gluing $E_{1}$ and $E_{2}$ together along their 2-torsion subgroups gives us a genus- 2 curve $C$ over $\mathbb{F}_{p}$ with $10^{2013}$ points. Carrying out this procedure and cleaning up the resulting equations as much as possible, we obtain the following result.

THEOREM 7.1. Let $p$ be the 2014-digit prime specified in the preceding paragraph, and let $u$ be any one of the three elements of $\mathbb{F}_{p}$ that satisfies $u^{3}+u+1=0$. Then the genus- 2 curve $C / \mathbb{F}_{p}$ defined by

$$
y^{2}=(u-1)\left(x^{2}+8\right)\left(x^{4}+16 x^{2}+u^{24}\right)
$$

has exactly $10^{2013}$ rational points.

Magma code verifying this example can be found at the URL mentioned above.

\subsection{A genus-2 curve of order $10^{2014}+9703$}

Again, to produce a genus- 2 curve of order $N_{2}=10^{2014}+9703$, our algorithm requires that we start with an elliptic curve of order $N_{2}$. The Bröker-Stevenhagen algorithm produced an elliptic curve $E_{1}$ over a field $\mathbb{F}_{p}$, with End $E_{1}$ the quadratic order of discriminant $-96097 \cdot 127363$. Producing the curve $E_{1}$ required finding a root in $\mathbb{F}_{p}$ of a class polynomial for this quadratic order; we thank Andrew Sutherland for carrying out the computation for us, using the methods of $[24]$. 
Since $N_{2}$ is odd, we must take $\ell=3$ in Algorithm 6.8. We compute that the curve $E_{1}$ is minimal at $\ell$.

The prime $p$ is congruent to -1 modulo 7 , so $p$ is inert in the quadratic field $\mathbb{Q}(\sqrt{-7})$, and hence the elliptic curve $E_{2}$ over $\mathbb{F}_{p}$ defined by $y^{2}=x^{3}-35 x+98$, which has CM by the order of discriminant -7 , is supersingular and has trace zero. Applying Algorithm 5.4, we find a genus-2 curve $C$ with degree-3 maps to both $E_{1}$ and $E_{2}$, and this $C$ therefore has exactly $N_{2}$ rational points. Equations for $E_{1}$ and $C$ can be found at the URL mentioned above.

Note that even though the input $N_{2}$ is a number that we did not prove to be prime, the output of our algorithm is correct if the input is; that is, if $N_{2}$ is indeed prime. Actually, the fact that Algorithm 6.8 produces any output at all is already a strong probabilistic proof of the primality of $N_{2}$, because the Bröker-Stevenhagen subroutine in Step 2 requires the computation of a large number of square roots of potential discriminants $\Delta$ modulo $N_{2}$ in order to succeed.

\section{Appendix A. Genus-2 triple covers of elliptic curves}

As we noted in $\S 5$, explicit families of genus- 2 curves with degree- 3 maps to elliptic curves appeared in the literature over 125 years ago. Indeed, in addition the family of curves given by Goursat [8] in 1885, which includes, with a single exception, every genus- 2 curve over $\mathbb{C}$ with a degree-3 map to an elliptic curve, there is also an 1876 paper of Hermite [9] that gives formulas for the one-parameter family of triple covers $C \rightarrow E$ over $\mathbb{C}$ called 'special' by Kuhn [16] and 'degenerate' by Shaska [22], and that includes the curve missed by Goursat's family.

However, neither these nineteenth-century works nor their modern counterparts provide exactly what we would like to have: a complete parametrization, over an arbitrary base field $k$, of the family of genus- 2 curves over $k$ that have $k$-rational degree- 3 maps to elliptic curves, including formulas for the genus- 2 curves, the associated elliptic curves, and the degree- 3 maps. In this appendix we provide such parametrizations, the sole restriction being that we assume the characteristic of $k$ is neither two nor three. The family of genus-2 curves we obtain is essentially identical to that of Goursat [8, Exemple II, pp. 155-157].

\section{A.1. The parametrization}

We start by writing down a family of curves and maps. Let $k$ be a field of characteristic neither two nor three, and let $a, b, c, d, t$ be elements of $k$ satisfying

$$
12 a c+16 b d=1, \quad a^{3}+b^{2} \neq 0, \quad c^{3}+d^{2} \neq 0, \quad t \neq 0 .
$$

Set $\Delta_{1}=a^{3}+b^{2}$ and $\Delta_{2}=c^{3}+d^{2}$, and define polynomials $f, f_{1}, f_{2}$ by

$$
\begin{aligned}
f & =\left(x^{3}+3 a x+2 b\right)\left(2 d x^{3}+3 c x^{2}+1\right), \\
f_{1} & =x^{3}+12\left(2 a^{2} d-b c\right) x^{2}+12\left(16 a d^{2}+3 c^{2}\right) \Delta_{1} x+512 \Delta_{1}^{2} d^{3}, \\
f_{2} & =x^{3}+12\left(2 b c^{2}-a d\right) x^{2}+12\left(16 b^{2} c+3 a^{2}\right) \Delta_{2} x+512 \Delta_{2}^{2} b^{3} .
\end{aligned}
$$

Further, define rational functions $u_{1}, v_{1}, u_{2}, v_{2}$ by

$$
\begin{array}{ll}
u_{1}=12 \Delta_{1} \frac{-2 d x+c}{x^{3}+3 a x+2 b}, & v_{1}=\Delta_{1} \frac{16 d x^{3}-12 c x^{2}-1}{\left(x^{3}+3 a x+2 b\right)^{2}}, \\
u_{2}=12 \Delta_{2} \frac{x^{2}(a x-2 b)}{2 d x^{3}+3 c x^{2}+1}, & v_{2}=\Delta_{2} \frac{x^{3}+12 a x-16 b}{\left(2 d x^{3}+3 c x^{2}+1\right)^{2}} .
\end{array}
$$

The following lemma is purely computational, and we leave the proof to the reader and his or her computational algebra package. 
Lemma A.1. The discriminants of $f, f_{1}$, and $f_{2}$ are

$$
\begin{gathered}
\Delta(f)=2^{8} 3^{12} \Delta_{1}^{3} \Delta_{2}^{3}, \\
\Delta\left(f_{1}\right)=-2^{2} 3^{3} \Delta_{1}^{2} \Delta_{2}, \\
\Delta\left(f_{2}\right)=-2^{2} 3^{3} \Delta_{1} \Delta_{2}^{2} .
\end{gathered}
$$

Furthermore, for each $i=1,2$ there is a degree-3 morphism from the curve $t y^{2}=f$ to the curve $t y^{2}=f_{i}$ given by sending $(x, y)$ to $\left(u_{i}, y v_{i}\right)$.

The lemma shows that given $a, b, c, d, t$ in $k$ that satisfy (A.1), we obtain a genus-2 curve $C_{a, b, c, d, t}$ defined by $t y^{2}=f$, two elliptic curves $E_{a, b, c, d, t, 1}$ and $E_{a, b, c, d, t, 2}$ defined by $t y^{2}=f_{1}$ and $t y^{2}=f_{2}$, and degree-3 maps

$$
\begin{aligned}
& \varphi_{a, b, c, d, t, 1}: C_{a, b, c, d, t} \rightarrow E_{a, b, c, d, t, 1} \\
& (x, y) \mapsto\left(u_{1}, y v_{1}\right) \\
& \begin{aligned}
\varphi_{a, b, c, d, t, 2}: C_{a, b, c, d, t} & \rightarrow E_{a, b, c, d, t, 2} \\
(x, y) & \mapsto\left(u_{2}, y v_{2}\right) .
\end{aligned}
\end{aligned}
$$

It is easy to choose values of $a, b, c, d$, and $t$ in $\mathbb{Q}$ so that the curves $E_{a, b, c, d, t, 1}$ and $E_{a, b, c, d, t, 2}$ are geometrically nonisogenous. Therefore, for generic values of $a, b, c, d, t$, the morphism

$$
\varphi_{a, b, c, d, t, 2 *} \varphi_{a, b, c, d, t, 1}^{*}: E_{a, b, c, d, t, 1} \rightarrow E_{a, b, c, d, t, 2}
$$

is the zero map, so it must be the zero map for all values of $a, b, c, d, t$ in any field. It follows that Jac $C_{a, b, c, d, t}$ is isogenous to the product of $E_{a, b, c, d, t, 1}$ and $E_{a, b, c, d, t, 2}$.

Note that if $\lambda$ and $\mu$ are elements of $k^{*}$, then scaling $x$ by $\lambda$ and $y$ by $\mu$ in the equations for these curves and maps is equivalent to replacing the quintuple $(a, b, c, d, t)$ with $\left(\lambda^{2} a, \lambda^{3} b, \lambda^{-2} c, \lambda^{-3} d, \lambda \mu^{2} t\right)$. This gives an action of $k^{*} \times k^{*}$ on the set of quintuples. Note that one can always scale a quintuple by this action in order to obtain $t=1$.

Proposition A.2. Let $k$ be a field of characteristic neither two nor three. Suppose that $\varphi_{1}: C \rightarrow E_{1}$ and $\varphi_{2}: C \rightarrow E_{2}$ are degree-3 maps from a genus-2 curve $C$ over $k$ to genus1 curves $E_{1}$ and $E_{2}$ over $k$, and suppose that the morphism $\varphi_{2 *} \varphi_{1}^{*}$ from Jac $E_{1}$ to Jac $E_{2}$ is the zero map. Then there are elements $a, b, c, d, t$ of $k$ satisfying (A.1) and isomorphisms $\alpha: C \rightarrow C_{a, b, c, d, t}$ and $\alpha_{i}: E_{i} \rightarrow E_{a, b, c, d, t, i}$ such that the diagram

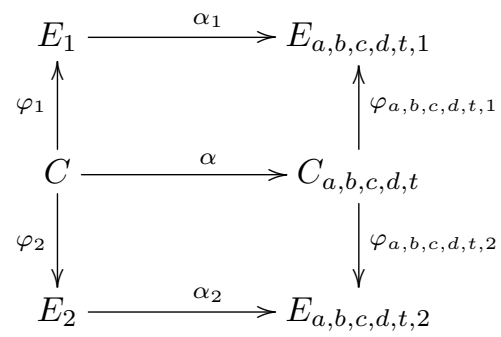

commutes. The quintuple $(a, b, c, d, t)$ is unique up to the action of $k^{*} \times k^{*}$ given above.

The following lemma will be helpful in our proof of the proposition.

Lemma A.3. Suppose that $\varphi: C \rightarrow E$ and $\psi: C \rightarrow F$ are degree-3 maps from a curve $C$ to genus-1 curves $E$ and $F$ over a field $k$. If $\varphi$ and $\psi$ have the same ramification divisor, then there is an isomorphism $\alpha: E \rightarrow F$ such that $\psi=\alpha \varphi$. 
Remark A.4. The argument given by Kuhn [16, Corollary, p. 45] shows that $E$ and $F$ both have rational points, so they can be made into elliptic curves. We phrase the lemma and the proposition in terms of genus- 1 curves because the isomorphism $\alpha$ in the lemma, and the isomorphisms $\alpha_{1}$ and $\alpha_{2}$ in the proposition, may not be morphisms of elliptic curves - they do not necessarily take the identity element of one curve to the identity element of the other.

Proof of Lemma A.3. Let $\omega_{E}$ and $\omega_{F}$ be nonzero holomorphic differentials on $E$ and $F$. The pullbacks $\varphi^{*} \omega_{E}$ and $\psi^{*} \omega_{F}$ are holomorphic differentials on $C$, and the divisors of these differentials are the ramification divisors of the maps $\varphi$ and $\psi$. Since the ramification divisors are equal by assumption, the two pullbacks differ by a multiplicative constant.

Let $\bar{k}$ be the algebraic closure of $k$, and let $C_{\bar{k}}$ and $J_{\bar{k}}$ be the base extensions of $C$ and its Jacobian $J$ to $\bar{k}$. One can embed $C_{\bar{k}}$ into $J_{\bar{k}}$, and the embedding induces an isomorphism from the holomorphic differentials on $J_{\bar{k}}$ to the holomorphic differentials on $C_{\bar{k}}$ (see [21, Proposition 2.2]). This shows that the pullbacks of $\omega_{E}$ and $\omega_{F}$ to $J$ also differ by a constant, so that the images of $E$ and $F$ in $J$ are the same. But since the degrees of $\varphi$ and $\psi$ are prime, $E$ and $F$ are isomorphic to their images in $J$. The induced isomorphism $\alpha: E \rightarrow F$ then satisfies $\psi=\alpha \varphi$.

Proof of Proposition A.2. The Riemann-Hurwitz formula shows that the map $\varphi_{1}$ is ramified either at two points, with ramification index two (Shaska's 'nondegenerate' case, and Kuhn's 'generic' case), or at one point, with ramification index three (Shaska's 'degenerate' case, and Kuhn's 'special' case). Kuhn [16, Lemma, p. 42] shows that in the former case the two ramification points are conjugate with respect to the hyperelliptic involution, and that in the latter case the single ramification point is a Weierstrass point. Let $\mathbb{P}_{C}^{1}$ be the quotient of $C$ by the hyperelliptic involution. We can choose a parameter $x$ on $\mathbb{P}_{C}^{1}$ so that the $x$-coordinate of the ramification points (or point) is equal to zero.

Suppose that we are in Kuhn's generic case. Kuhn $[\mathbf{1 6}, \S 6]$ shows that then $C$ has a model of the form

$$
y^{2}=\left(x^{3}+\ell x^{2}+m x+n\right)\left(4 n x^{3}+m^{2} x^{2}+2 m n x+n^{2}\right),
$$

where $n \neq 0$, and that the ramification point(s) of the map $\varphi_{2}$ then have $x$-coordinate equal to $-3 n / m$.

If we apply a linear fractional transformation that takes zero to $\infty$ and $-3 n / m$ to 0 , we find that the model for $C$ transforms to a curve of the form $C_{a, b, c, d, t}$. Furthermore, the ramification points of the maps $\varphi_{1}$ and $\varphi_{2}$ are the same as the ramification points for $\varphi_{a, b, c, d, t, 1}$ and $\varphi_{a, b, c, d, t, 2}$, so by Lemma A.3, there are isomorphisms $\alpha, \alpha_{1}$, and $\alpha_{2}$ as in the statement of the proposition so that the diagram in the proposition is commutative.

Now suppose that $\varphi_{1}$ is degenerate, in Shaska's terminology. Arguing as in [22, § 2.2], but keeping track of fields of definition, we find that by moving the $x$-coordinate of the ramification point of $\varphi_{1}$ to $\infty$ and by translating and scaling $x$ appropriately, we can write $C$ as

$$
y^{2}=\left(3 x^{2}+4 m\right)\left(x^{3}+m x+n\right),
$$

where $m \neq 0$; then we compute that the ramification point(s) of the map $\varphi_{2}$ have $x$-coordinate equal to 0. Once again, by applying Lemma A.3, we find that there are isomorphisms $\alpha$, $\alpha_{1}$, and $\alpha_{2}$ as in the statement of the proposition so that the diagram in the proposition is commutative.

To complete the proof, we must show that the quintuple $(a, b, c, d, t)$ is unique up to the action of $k^{*} \times k^{*}$. We obtained our model $C_{a, b, c, d, t}$ for the curve $C$ by taking two marked points on $\mathbb{P}_{C}^{1}$-namely, the $x$-coordinates of the ramification points of the maps $\varphi_{1}$ and $\varphi_{2}-$ and moving them to $\infty$ and 0 , respectively. That choice determines the parameter $x$ of $\mathbb{P}_{C}^{1}$ up to a scaling factor. But the action of $k^{*} \times k^{*}$ on quintuples $(a, b, c, d, t)$ is exactly the action obtained from scaling the coordinates $x$ and $y$ for $C_{a, b, c, d, t}$. 


\section{A.2. Additional formulas}

We compute that the $j$-invariants of the elliptic curves $E_{a, b, c, d, t, 1}$ and $E_{a, b, c, d, t, 2}$ are given by

$$
\begin{aligned}
& j\left(E_{a, b, c, d, t, 1}\right)=\frac{1728\left(a^{2} c+4 a b d-4 b^{2} c^{2}\right)^{3}}{\Delta_{1}^{2} \Delta_{2}}, \\
& j\left(E_{a, b, c, d, t, 2}\right)=\frac{1728\left(a c^{2}+4 b c d-4 a^{2} d^{2}\right)^{3}}{\Delta_{1} \Delta_{2}^{2}} .
\end{aligned}
$$

We use these $j$-invariant formulas in Algorithm 5.4.

Let $\omega_{1}$ and $\omega_{2}$ be the invariant differentials $d x / 2 y$ on $E_{a, b, c, d, t, 1}$ and $E_{a, b, c, d, t, 2}$, respectively. It is not hard to verify that then we have

$$
\varphi_{a, b, c, d, t, 1}^{*} \omega_{1}=\frac{3 d x}{2 y} \quad \text { and } \quad \varphi_{a, b, c, d, t, 2}^{*} \omega_{2}=\frac{3 x d x}{2 y}
$$

on the curve $C_{a, b, c, d, t}$.

\section{A.3. A note on degeneration}

Note that the map $\varphi_{a, b, c, d, t, 1}$ is special (in Kuhn's terminology) exactly when $d=0$, and that the map $\varphi_{a, b, c, d, t, 2}$ is special exactly when $b=0$. We close this appendix by explaining why our formulas degenerate nicely to these special cases, whereas the formulas of Kuhn and Shaska do not.

Let $\varphi_{1}$ and $\varphi_{2}$ be as above. As Kuhn [16, Lemma, p. 42] notes, the hyperelliptic involution on $C$ descends via $\varphi_{1}$ to an involution on $E_{1}$ that gives a degree- 2 map from $E_{1}$ to a projective line $\mathbb{P}_{E_{1}}^{1}$. Then $\varphi_{1}$ induces a degree-3 map $\varphi_{1}^{\prime}$ from $\mathbb{P}_{C}^{1}$ to $\mathbb{P}_{E_{1}}^{1}$.

Suppose that $\varphi_{1}$ is generic. Then the two ramification points $P_{1}$ and $Q_{1}$ of $\varphi_{1}$ share the same image $x_{1}$ in $\mathbb{P}_{C}^{1}$, and $x_{1}$ is doubly ramified in the triple cover $\varphi_{1}^{\prime}$. Let $y_{1}$ be the other point of $\mathbb{P}_{C}^{1}$ with $\varphi_{1}^{\prime}\left(y_{1}\right)=\varphi_{1}^{\prime}\left(x_{1}\right)$.

The special maps are the limiting cases that occur when $P_{1}$ and its involute $Q_{1}$ approach a Weierstrass point of $C$. When $P_{1}=Q_{1}$, the point $x_{1}$ of $\mathbb{P}_{C}^{1}$ is triply ramified in $\varphi_{1}^{\prime}$, so the special maps can also be viewed as the limiting cases when $y_{1}$ approaches $x_{1}$.

Both Kuhn and Shaska choose their parametrizations of generic triple covers $C \rightarrow E_{1}$ so that the points $x_{1}$ and $y_{1}$ lie at 0 and $\infty$. Since the special triple covers have $x_{1}=y_{1}$, the parametrizations of Kuhn and Shaska cannot degenerate gracefully.

We have chosen our parametrization so that $x_{1}=\infty$ and so that the corresponding point $x_{2}$ from the cover $\varphi_{2}$ lies at 0 . Since Lemma A.3 shows that $x_{1}$ and $x_{2}$ can never be equal, there is no reason for the parametrization to break down at the special covers.

\section{References}

1. R. C. BAker, G. Harman and J. Pintz, 'The difference between consecutive primes. II', Proc. Lond. Math. Soc. (3) 83 (2001) no. 3, 532-562; doi:10.1112/plms/83.3.532.

2. R. Bröker, 'Constructing supersingular elliptic curves', J. Comb. Number Theory 1 (2009) no. 3, $269-273$.

3. R. Bröker and P. Stevenhagen, 'Efficient CM-constructions of elliptic curves over finite fields', Math. Comp. 76 (2007) no. 260, 2161-2179; doi:10.1090/S0025-5718-07-01980-1.

4. H. Cohen, F. Diaz y Diaz and M. Olivier, 'Enumerating quartic dihedral extensions of $\mathbb{Q}$ ', Compositio Math. 133 (2002) no. 1, 65-93; doi:10.1023/A:1016310902973.

5. K. EISEntrÄGER and K. LAUter, 'A CRT algorithm for constructing genus 2 curves over finite fields', Arithmetics, geometry and coding theory (AGCT 2005), Séminaires et Congrés 21 (eds F. Rodier and S. Vlăduţ; Société Mathématique de France, Paris, 2010) 161-176.

6. M. Fouquet and F. Morain, 'Isogeny volcanoes and the SEA algorithm', Algorithmic number theory (Sydney, 2002), Lecture Notes in Computer Science 2369 (eds C. Fieker and D. R. Kohel; Springer, Berlin, 2002) 276-291; doi:10.1007/3-540-45455-1_23. 
7. G. Frey and E. KANI, 'Curves of genus 2 covering elliptic curves and an arithmetical application', Arithmetic algebraic geometry (Texel, 1989), Progress in Mathematics 89 (eds G. van der Geer, F. Oort and J. H. M. Steenbrink; Birkhäuser, Boston, 1991) 153-176; doi:10.1007/978-1-4612-0457-2_7.

8. E. Goursat, 'Sur la réduction des intégrales hyperelliptiques', Bull. Soc. Math. France 13 (1885) 143-162; http://www.numdam.org/item?id=BSMF_1885__13__143_1.

9. Ch. Hermite, 'Sur un exemple de réduction d'intégrales abéliennes aux fonctions elliptiques', Ann. Soc. Sci. Bruxelles Sér. I 1, 2nd part (1876) 1-16; http://books.google.com/books?id=gAvjAX4hTeYC.

10. E. W. Howe, F. Leprévost and B. Poonen, 'Large torsion subgroups of split Jacobians of curves of genus two or three', Forum Math. 12 (2000) no. 3, 315-364; doi:10.1515/form.2000.008.

11. E. W. Howe, E. Nart and C. Ritzenthaler, 'Jacobians in isogeny classes of abelian surfaces over finite fields', Ann. Inst. Fourier (Grenoble) 59 (2009) no. 1, 239-289; doi:10.5802/aif.2430.

12. C. G. J. JACOBI, 'Review of Legendre's Traité des fonctions elliptiques, troisième supplément', J. reine angew. Math. 8 (1832) 413-417; http://resolver.sub.uni-goettingen.de/purl?PPN243919689_0008.

13. C. G. J. JACOBI, Gesammelte Werke, Bände I-VIII (Chelsea, New York, 1969).

14. E. KANI, 'The number of curves of genus two with elliptic differentials', J. reine angew. Math. 485 (1997) 93-121; doi:10.1515/crll.1997.485.93.

15. D. R. Kohel, 'Endomorphism rings of elliptic curves over finite fields', $\mathrm{PhD}$ Thesis, University of California, Berkeley, 1996.

16. R. M. Kunn, 'Curves of genus 2 with split Jacobian', Trans. Amer. Math. Soc. 307 (1988) no. 1, 41-49; doi: $10.2307 / 2000749$.

17. A. M. Legendre, Traité des fonctions elliptiques et des intégrales Eulériennes, Tome troisième (HuzardCourcier, Paris, 1828); http://gallica.bnf.fr/ark:/12148/bpt6k110149h.

18. S. R. Louboutin, 'Lower bounds for relative class numbers of imaginary abelian number fields and CM-fields', Acta Arith. 121 (2006) no. 3, 199-220; doi:10.4064/aa121-3-1.

19. К. Матомёкі, 'Large differences between consecutive primes', Q. J. Math. 58 (2007) no. 4, 489-518; doi:10.1093/qmath/ham021.

20. J.-F. Mestre, 'Construction de courbes de genre 2 à partir de leurs modules', Effective methods in algebraic geometry (Castiglioncello, 1990), Progress in Mathematics 94 (eds T. Mora and C. Traverso; Birkhäuser, Boston, 1991) 313-334; doi:10.1007/978-1-4612-0441-1_21.

21. J. S. Milne, 'Jacobian varieties', Arithmetic geometry (Storrs, Conn., 1984) (eds G. Cornell and J. H. Silverman; Springer, New York, 1986) 167-212; http://jmilne.org/math/articles/1986c.pdf.

22. T. Shaska, 'Genus 2 fields with degree 3 elliptic subfields', Forum Math. 16 (2004) no. 2, 263-280; doi:10.1515/form.2004.013.

23. M. Streng, 'Computing Igusa class polynomials', Math. Comp. 83 (2014) no. 285, 275-309; doi:10.1090/S0025-5718-2013-02712-3.

24. A. V. Sutherland, 'Accelerating the CM method', LMS J. Comput. Math. 15 (2012) 172-204; doi:10.1112/S1461157012001015.

25. J. TAte, 'Classes d'isogénie des variétés abéliennes sur un corps fini (d'après T. Honda)', Séminaire Bourbaki, Vol. 1968/69: Exposés 347-363, Lecture Notes in Mathematics 179 (Springer, Berlin, 1971) 95-110; doi:10.1007/BFb0058807.

\section{Reinier Bröker}

Department of Mathematics

Brown University

Box 1917, 151 Thayer Street

Providence

RI 02912, USA

reinier@math.brown.edu

\author{
Kristin E. Lauter \\ Microsoft Research \\ One Microsoft Way \\ Redmond \\ WA 98052, USA
}

klauter@microsoft.com

\author{
Everett W. Howe \\ Center for Communications Research \\ 4320 Westerra Court \\ San Diego \\ CA 92121, USA
}

however@alumni.caltech.edu

Peter Stevenhagen
Mathematisch Instituut
Universiteit Leiden
Postbus 9512
2300 RA Leiden
The Netherlands
psh@math.leidenuniv.nl 